\title{
The mystery behind the mid-temperature transition(s) in vinylidenefluoride-based homo-, co- and terpolymers - Has the puzzle been solved?
}

\author{
Thulasinath Raman Venkatesan \\ University of Potsdam, Institute of Physics and Astronomy \\ Karl-Liebknecht-Strasse 24-25,14476 Potsdam, Germany \\ and \\ KU Leuven, Department of Physics and Astronomy \\ Celestijinenlaan 200D, 3001 Leuven, Belgium \\ Michael Wübbenhorst \\ KU Leuven, Department of Physics and Astronomy \\ Celestijinenlaan 200D, 3001 Leuven, Belgium \\ Bernd Ploss \\ University of Applied Sciences Jena, Department of SciTec \\ Carl-Zeiss-Promenade 2, 07745 Jena, Germany \\ Xunlin Qiu \\ Technical University of Chemnitz, Institute for Print and Media Technology \\ Reichenhainer Straße 70, 09126 Chemnitz, Germany \\ Takashi Nakajima \\ Tokyo University of Science, Department of Applied Physics \\ 6-3-1 Niijuku, Katsushika-ku, 125-8585 Tokyo, Japan

\section{Takeo Furukawa} \\ Kobayasi Institute of Physical Research \\ 3-20-41 Higashi-Motomachi, Kokubunji , 185-0022 Tokyo, Japan

\section{and Reimund Gerhard} \\ University of Potsdam, Institute of Physics and Astronomy \\ Karl-Liebknecht-Strasse 24-25, 14476 Potsdam, Germany
}

\begin{abstract}
Over the last half century, the existence of an additional thermal transition in between the glass transition and the Curie/melting transition has been frequently observed on vinylidenefluoride-based ferro-, pyro- and piezoelectric homo- and co-polymers. The transition has also been observed recently in some of the related relaxor-ferroelectric terpolymers. Despite its well-known existence and the rich history of its treatment in the literature, the origin(s) and a more or less complete picture of the mid-temperature transition have remained elusive until now. Over the years, several authors have put forth various explanations for the so-called mid-temperature transition - some complementary and some contradictory to each other. At the 17th IEEE International Symposium on Electrets (ISE-17) in Limerick, Ireland, in September 2019, the mysterious mid-temperature transition and its possible mechanism(s) became the subject of a panel discussion a) to mark the Golden Jubilee of the discovery of piezoelectricity in Polyvinylidenefluoride (PVDF) by Heiji Kawai of Kobayashi Institute of Physical Research, Japan, as well as the Centennial of the first recognition of ferroelectricity in
\end{abstract}


piezoelectric Seignette's or Rochelle salt. The panel put forward a new hypothesis that the mid-temperature transition is most likely a result of several interrelated processes that take place within the respective temperature range. The relevant processes include an upper glass transition or relaxation, a relaxation related to conformational disorder, possible imperfect/time-dependent structures formed as a result of thermal processing and secondary crystallization, as well as interface polarization effects at crystallineamorphous boundaries. The article captures the essence of the panel discussion and the perspectives obtained therefrom to elucidate the complex mid-temperature transition in vinylidenefluoride-based ferro-, pyro- and piezoelectric homo-, co- and ter-polymers.

Index Terms — vinylidenefluoride-based polymers, ferro-, pyro- and piezoelectric polymers, mid-temperature transition, dielectric relaxation spectroscopy, nonlinear dielectric spectroscopy, differential scanning calorimetry

\section{INTRODUCTION}

Since the discovery of piezoelectricity in poly(vinylidene fluoride) (PVDF) fifty years ago by Heiji Kawai in Japan [1], the relevant properties of the homopolymer PVDF and of various co- and ter-polymers with VDF have attracted considerable interest. While pyroelectricity was already known in ancient Greece [2] and probably in China more than two thousand years ago, piezoelectricity had only been discovered in France by Jacques and Pierre Curie in 1880 on tourmaline, quartz, Seignette's (or Rochelle) salt and other crystals [3] and quickly became a "hot topic" [4]. On piezoelectric Rochelle salt, ferroelectricity was finally recognized and described by Joseph Valasek one hundred years ago in 1920 [5]. At the same time, i.e. in the year 1920, synthetic polymers were first postulated by Hermann Staudinger [6], but piezoelectricity in natural and synthetic polymers was only investigated by Eiichi Fukada in Japan in the 1950s and 1960s [7]. Soon after the discovery of piezoelectricity in PVDF [1], the related properties of pyroelectricity and optical second-harmonic generation in PVDF were reported by a team from Bell Laboratories [8]. In several studies mainly by a Japanese research team around Eiichi Fukada and Takeo Furukawa, ferroelectricity in PVDF was clearly demonstrated between 1975 and 1980 [9].

Nevertheless, the origin of the unexpected ferroelectric and electret properties in PVDF was debated quite intensively - in particular the question whether charges or dipoles or both were essential for the observed piezo-, pyro- and ferroelectric phenomena. The discussion about charges and/or dipoles had

Manuscript received on xx Month 20yy, in final form xx Month 20yy, accepted xx Month 20yy. Corresponding author: Thulasinath Raman Venkatesan Email: thulasinath.raman.venkatesan@uni-potsdam.de

a) The Panel Discussion was proposed and organized by Thulasinath Raman Venkatesan on the invitation of the organizers of the $17^{\text {th }}$ IEEE International Symposium on Electrets (ISE-17) in September 2019. Bernd Ploss moderated the Panel Discussion at ISE-17. The panelists Thulasinath Raman Venkatesan (T.R.V.) (section 2), Michael Wübbenhorst (M.W) (section 3), Takash Nakajima (T.N.) (section 4), Xunlin Qiu (X.Q.) (section 5) and Bernd Ploss (B.P.) (section 6) presented their respective data and perspectives on the observed transition. Important contributions were made by Takeo Furukawa (section 7) who was a member of the audience. Interactive discussions between the panelists and the members of the audience were transcribed by T.R.V. to provide a basis for the present review on the invitation of the editors of the ISE special issue. The manuscript has been co-written, edited and revised by all authors. The project of such a discussion and the preparation and revision of the manuscript were supported and guided by Reimund Gerhard and Takeo Furukawa. accompanied modern experimental electret research since its beginnings one century ago with the seminal work on carnaubawax electrets started by Mototarô Eguchi in 1919 [10] and summarised in 1925 [11]. The observation of charges and dipoles and their interaction on and in electrets led to the introduction of the concept of hetero-charge (internal space charge and dipole charges; polarity opposite to the polarity of the adjacent electrode) and homo-charge (injected charge; the same polarity as that of the adjacent electrode) [12]. With respect to PVDF, it was proposed that the orientation of dipoles in the crystalline $\beta$ phase was necessary for piezoelectricity but only for providing the trapping sites for the charges that caused the polarisation component which leads to piezo- and pyroelectricity [13-15] . Today, it is instead accepted that the ferro-, pyro- and piezoelectricity of PVDF and related polymers originate from the polarisation of its ordered crystallites, but also that the amorphous phase around the crystallites and the interface charges at crystalline-amorphous "boundaries" can play significant roles in the piezoelectric response and its stabilisation [16-18] .

The complexity of PVDF and its copolymers with trifluoroethylene (TrFE), tetrafluoroethylene (TFE) and hexafluoropropylene (HFP) is not only a consequence of the interplay between molecular dipoles and internal plus injected charges, but also of a semi-crystalline morphology with up to five different crystalline modifications - some polar and some non-polar - and of several transitions and thus a strong influence of the respective thermal histories on a given polymer sample. The crystalline phases of PVDF [19] itself are usually designated as $\alpha, \beta, \gamma, \delta$ and $\boldsymbol{\varepsilon}$ with three different chain conformations that can be arranged with different dipole orientations: (I) all-trans or TTTT, a highly polar zig-zag chain, (II) trans-gauche or $T G^{+} T G^{-}$, a polar helical chain, and (III) trans-trans-trans-gauche or $T_{3} G^{+} T_{3} G^{-}$, a polar chain with alternating zig-zig and trans-gauche monomers ( $c f$. Figure 1). For historical reasons, the anti-parallel dipole arrangement of the $T G^{+} T G^{-}$chains is called $\alpha$-phase, as it is the non-polar phase that is formed upon crystallisation from the melt or from solution without further treatment. Upon stretching at a high ratio, the $\alpha$-phase may be transformed into the highly polar $\beta$ phase in which the TTTT chains are arranged with all dipoles in parallel. By means of high-field poling under suitable conditions, the $\alpha$-phase can be transformed into the polar $\alpha_{P}$ or $\delta$-phase that was discovered in $1977 / 78$ by Dilip Das-Gupta [20] and quickly confirmed by several other research groups. 
Thermal treatment (annealing) may convert the $T G^{+} T G^{-}$chains of the $\alpha$-phase into $T_{3} G^{+} T_{3} G^{-}$chains that may again be arranged either with their dipoles all in parallel (polar $\gamma$-phase) or with anti-parallel dipoles which constitute the non-polar $\boldsymbol{\varepsilon}$-phase discovered by Andrew Lovinger at Bell Laboratories in 1981/82 [21].

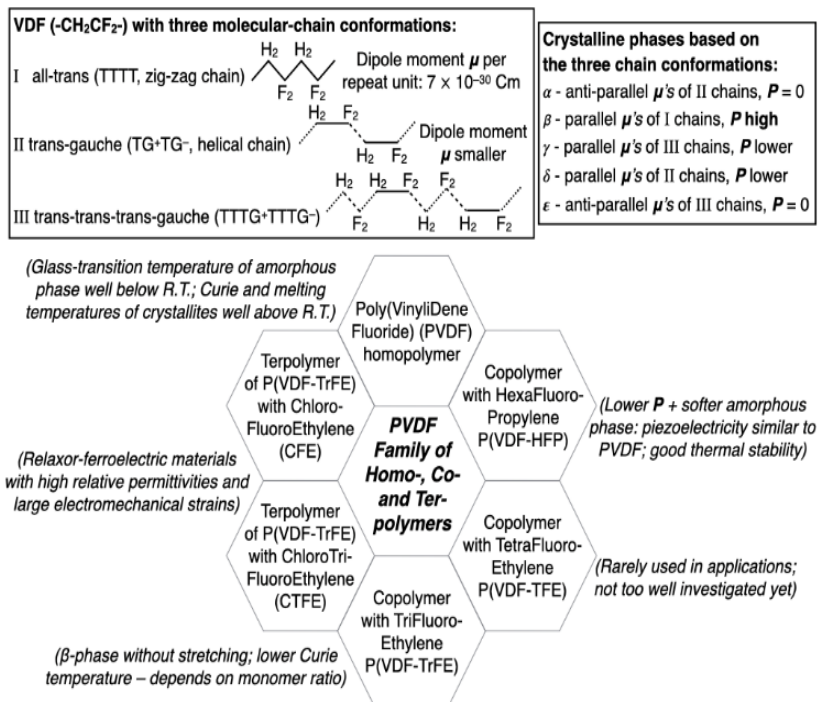

Figure 1. Schematic overview of the 3 chain conformations found in the 5 crystalline phases of vinylidene-fluoride (VDF)-based polymers and of the six most relevant VDF-based homo-, co- and ter-polymers with a few of their typical characteristics.

In the $\mathrm{P}$ (VDF-TrFE), $\mathrm{P}$ (VDF-TFE) and $\mathrm{P}$ (VDF-HFP) copolymers [22, 23], the VDF co-monomer segments are usually already found in the highly polar zig-zag conformation so that no further processing is required before electrical poling. For applications, however, the copolymers require optimisation of their monomer ratios with respect to the desired polymer properties in addition to the adjustment of other relevant parameters, as the maximum electric polarisation depends on VDF content and is stable only up to the Curie temperature at which the ferroelectric-to-paraelectric transition takes place. In the homopolymer PVDF, the Curie transition is masked by the melting process, a fact that contributed to the difficulties to establish ferroelectricity in PVDF. For P(VDF-TrFE) copolymers, however, the Curie temperature has to be optimised by adjusting the monomer ratio [22].

In 1985, Odajima and his colleagues from Hokkaido University in Japan reported that irradiation of P(VDF-TrFE) copolymers with gamma rays or high-energy electron beams led to a diffuse phase transition, i.e. to relaxor-ferroelectric behavior [24]. The discovery led to the development of relaxorferroelectric VDF-based copolymers with very high electrostrictive strains [25]. Soon after, an alternative route to relaxor-ferroelectricity in VDF-based copolymers was found: Addition of a third monomer, either chlorotrifluoroethylene (CTFE) [26] or chlorofluoroethylene (CFE) [27] (cf. Figure 1) also led to the required fragmentation of the VDF crystals into highly polar nano-domains and thus to the large dielectric permittivities and electromechanical responses that are typical of relaxor-ferroelectric polymers. In addition to the attractive electroactive properties of the relaxor-ferroelectric polymers, they have been prominently considered in strategies for designing dielectric polymers with high permittivity and low loss [28].

All the VDF-based polymers are frequently used in such applications as electromechanical actuation and transduction, electroacoustical sensing, electrocaloric refrigeration, dielectric energy storage, capacitive memory devices, etc. because of their ferro-, piezo- and pyroelectric properties. Though several detailed studies have been done in the past to understand the various phenomena and transitions observed in these materials, there are still uncertainties and open questions. Among them is the so-called mid-temperature transition, which is observed in all VDF-based homo-, co- and ter-polymers above room temperature, but usually well below the Curie and melting transitions. As the temperature range of the mid-temperature transition usually overlaps with the operating temperatures of the respective polymer devices, it is of importance not only for better understanding the underlying fundamental phenomena, but also for preventing undesired ageing and deterioration of the essential polymer materials in many applications. By means of dielectric, thermal and mechanical methods, several authors have tried to further explore and to understand the origin(s) of the transition since its first observation in the 1970s. While some researchers argue that it is a structural transition, others are strongly in favor of a relaxation. The contradicting views have further complicated the puzzle which still remains an open question.

Based on recent high-precision dielectric and thermal experiments, along with a review of relevant available literature on the topic, a new hypothesis is being put forward and discussed in the following. In section 2 below, it is attempted to explain the origin of the "mysterious" mid-temperature transition on the basis of detailed investigations. For the experimental research, the most often used ferroelectric polymers - polyvinylidene fluoride (PVDF), poly(vinylidene fluoride-trifluoroethylene) (P(VDF-TrFE)), poly(vinylidene fluoride-tetrafluoroethylene) (P(VDF-TFE) - and the relaxorferroelectric poly(vinylidene fluoride-trifluoroethylenechlorofluoroethylene) (P(VDF-TrFE-CFE) have been employed in the form of free-standing films (section 2). In the subsequent sections 3 through 8 , the new interpretation is amplified and discussed from different perspectives and with additional experimental evidence.

\section{THE ORIGINS BEHIND THE MID- TEMPERATURE TRANSITION IN VDF- BASED POLYMERS}

\subsection{PROSPOSED ORIGINS OF THE MID- TEMPERATURE TRANSITION - THE SITUATION}

Figure 2 shows the DSC thermograms of various VDF-based homo-, co- and ter-polymers during the first heating cycle. In addition to the glass-transition step which we see at low temperatures - and the Curie or the melting transitions which we see at higher temperatures, there exists a mid-temperature transition in between (red box in Figure 2). As seen in the Figure, it is very interesting to notice that irrespective of the 
processing condition, this transition appears in all VDF-based materials in the same temperature range, i.e. irrespective of whether the sample is stretched or unstretched, quenched or cooled slowly from the melt, annealed or non-annealed, the transition is found in all VDF-based materials. In spite (or because) of various explanations in the past, the origin of this transition has not completely or convincingly been explained. To begin with, in Figure 3, the permittivity and dielectric-loss spectra of an unstretched poly(vinylidene fluoridetetrafluroethylemne) $\mathrm{P}(\mathrm{VDF}-\mathrm{TFE})$ ) film is plotted as a function of frequency at selected temperatures. Clearly, we can identify two processes here: The one at higher frequencies, which is the conventional glass transition ( $\alpha_{\mathrm{a}^{-}}$or $\beta$-relaxation) process with peaks that shift with temperature, and the other one, which is the so-called mid-temperature $\left(T_{\text {mid }}\right)$ transition $\left(\alpha-\right.$ or $\alpha_{c^{-}}$ relaxation process) also showing temperature dependent peaks at low frequencies. In general, three major relaxations are observed in polymers. This includes the $\alpha$-relaxation process which involves the relaxation of dipolar units in the main chain of the polymer. So, it happens in both the crystalline and amorphous regions of the polymer and has the highest strength compared to the other molecular processes observed. When the relaxation occurs in the crystalline regions, it is sometimes referred to as $\alpha_{\mathrm{c}}$-relaxation. When the relaxation is associated

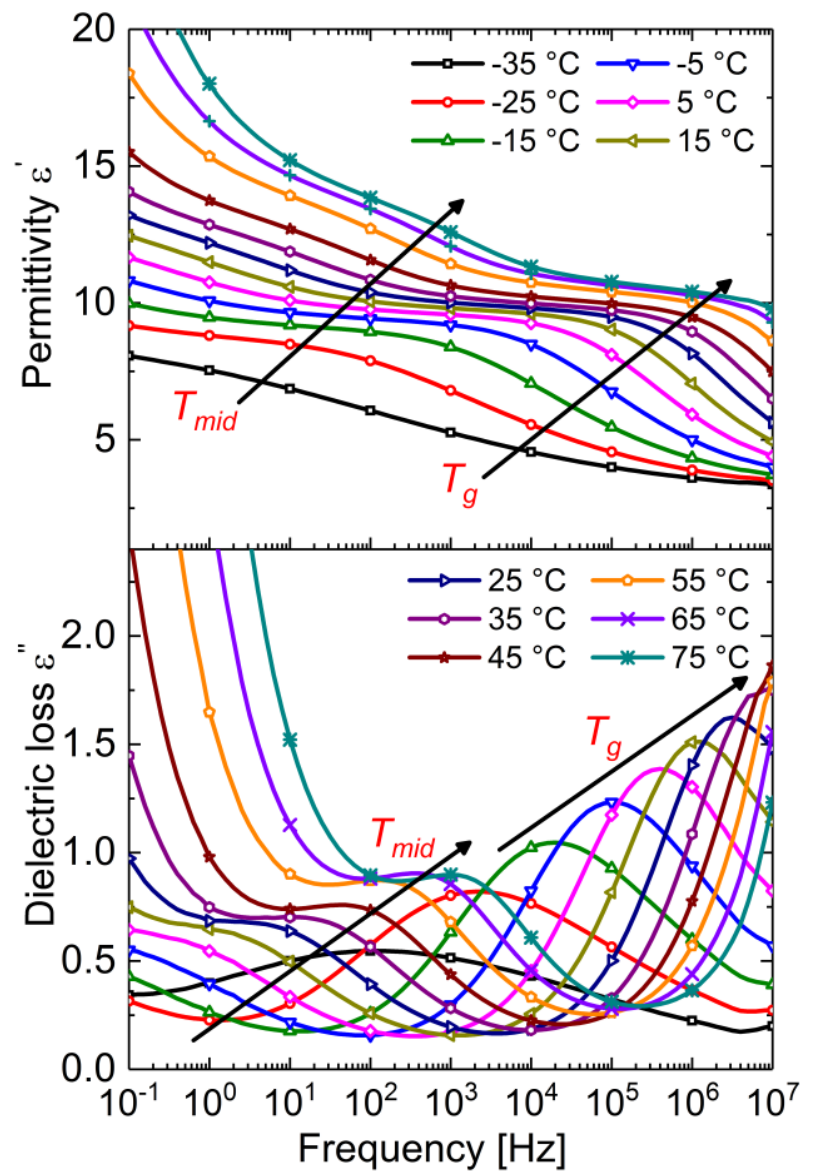

Figure 3. The permittivity and dielectric loss of an unstretched P(VDFTFE) film as a function of frequency from $10^{-1}$ to $10^{7} \mathrm{~Hz}$, plotted at selected temperatures. The curves were measured with 10 data points per frequency decade, and a few symbols were added to each curve in order to facilitate identification.

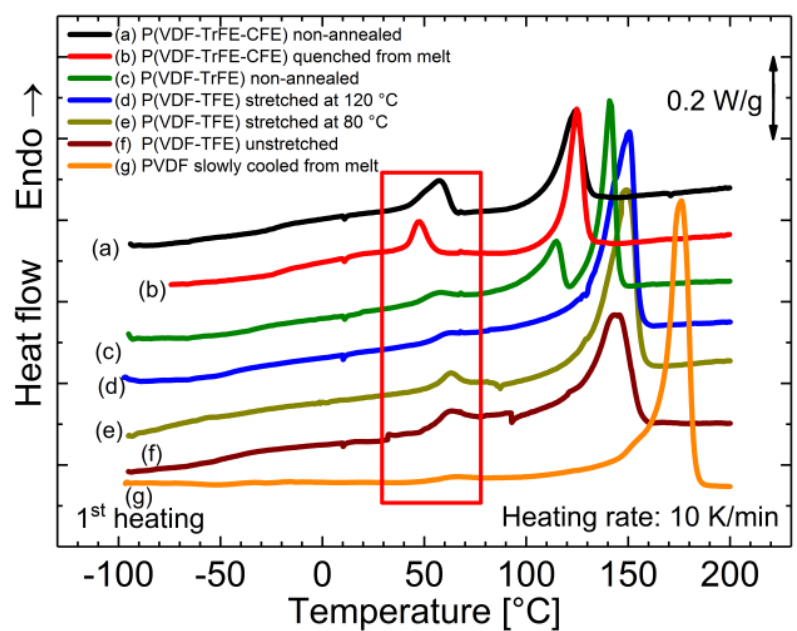

Figure 2. DSC thermograms of PVDF, P(VDF-TFE), P(VDF-TrFE) and $\mathrm{P}$ (VDF-TrFE-CFE) subjected to different heat treatments, recorded during the first heating run (heating rate of $10 \mathrm{~K} / \mathrm{min}$ ). The mid-temperature transition is found inside the red box in the center of the Figure.

with the micro-Brownian motions of the amorphous phase molecular segments, it is referred to as $\alpha_{\mathrm{a}}$-relaxation or $\beta$ relaxation process $[29,30]$. The $\beta$-process is associated with the structural transition of the polymer material from frozen glasslike state to mobile liquid-like state happening around the glass-

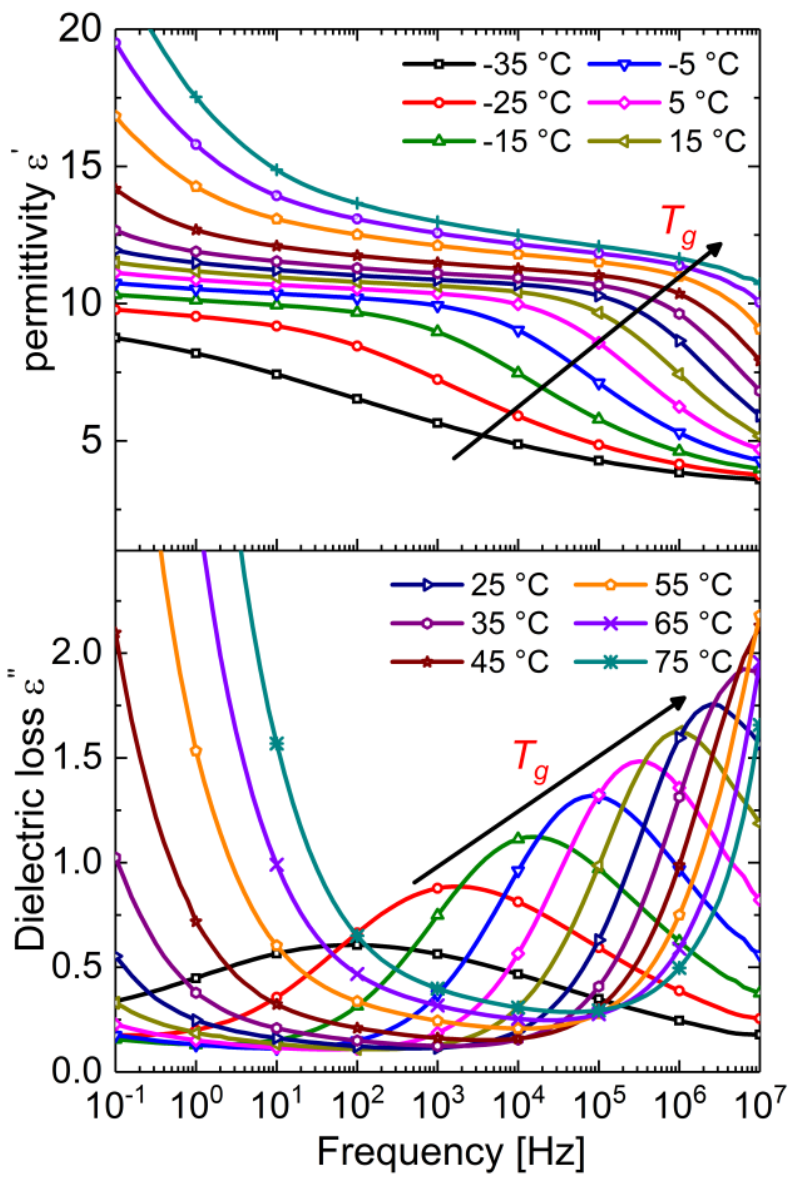

Figure 4. The permittivity and dielectric loss of a P(VDF-TFE) film stretched at a temperature of $80{ }^{\circ} \mathrm{C}$, plotted as a function of frequency from $10^{-1}$ to $10^{7} \mathrm{~Hz}$ at selected temperatures. The curves were measured with 10 data points per frequency decade, and a few symbols were added to each curve in order to facilitate identification. 
transition temperature $\left(T_{g}\right)$ of the polymer [29-31]. Other than these, the $\gamma$ relaxations occurring due to localized motions in the amorphous regions have also been observed [30]. Now,

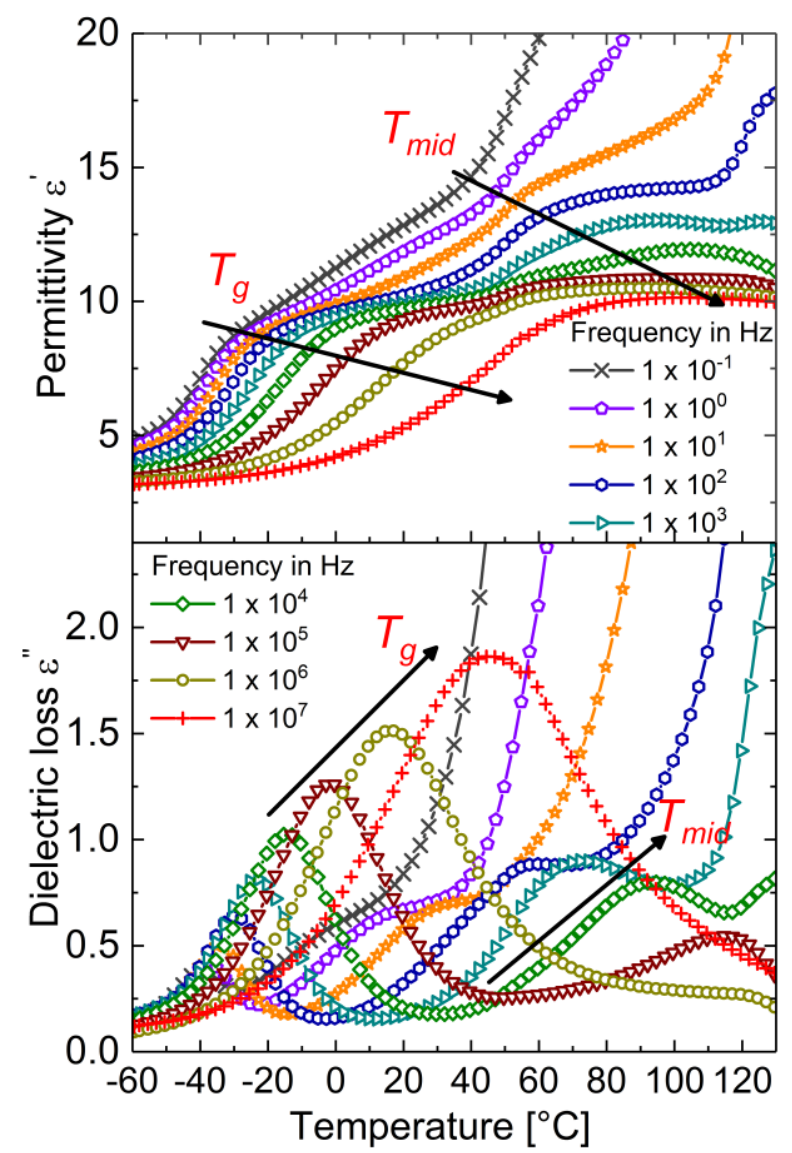

Figure 5. The permittivity and dielectric loss of an unstretched P(VDFTFE) film as a function of temperature at fixed frequencies from $10^{-1}$ to $10^{7} \mathrm{~Hz}$.

upon stretching, we can see that the $T_{\text {mid }}$ transition actually disappears -as shown in Figure 4. Therefore, both features, the temperature dependence of the peaks and their disappearance upon stretching -lead to the explanation that it could be because of the unfreezing of the amorphous regions that are constrained between crystallites. This is called an upper glass transition by certain authors [32-34]. Constrained amorphous phase refers to those amorphous regions which are embedded between crystallites [33, 35]. As their movement is restricted between crystalline regions, they do not undergo a conventional glasstransition at which the chains in continuous amorphous regions gain sufficient energy to move. The behavior is similar to that observed on XLPE [35]. Stretching removes these constrained regions which was stated as explanation for the absence of the transition in PVDF and P(VDF-TFE) samples measured immediately after stretching [33].

The same dielectric loss data of the unstretched P(VDF-TFE) sample shown in Figure 3 are plotted again in Figure 5, but now as a function of temperature instead of frequency. So, again, from the real and imaginary parts of the permittivity, we recognize both processes - namely, the glass transition at lower temperatures and the frequency-dependent mid-temperature transition above $0{ }^{\circ} \mathrm{C}$. However, the permittivity of a stretched
$\mathrm{P}(\mathrm{VDF}-\mathrm{TFE})$ sample, also plotted as a function of temperature in Figure 6, shows a small residue of the mid-temperature transition which is not observed in the frequency spectra

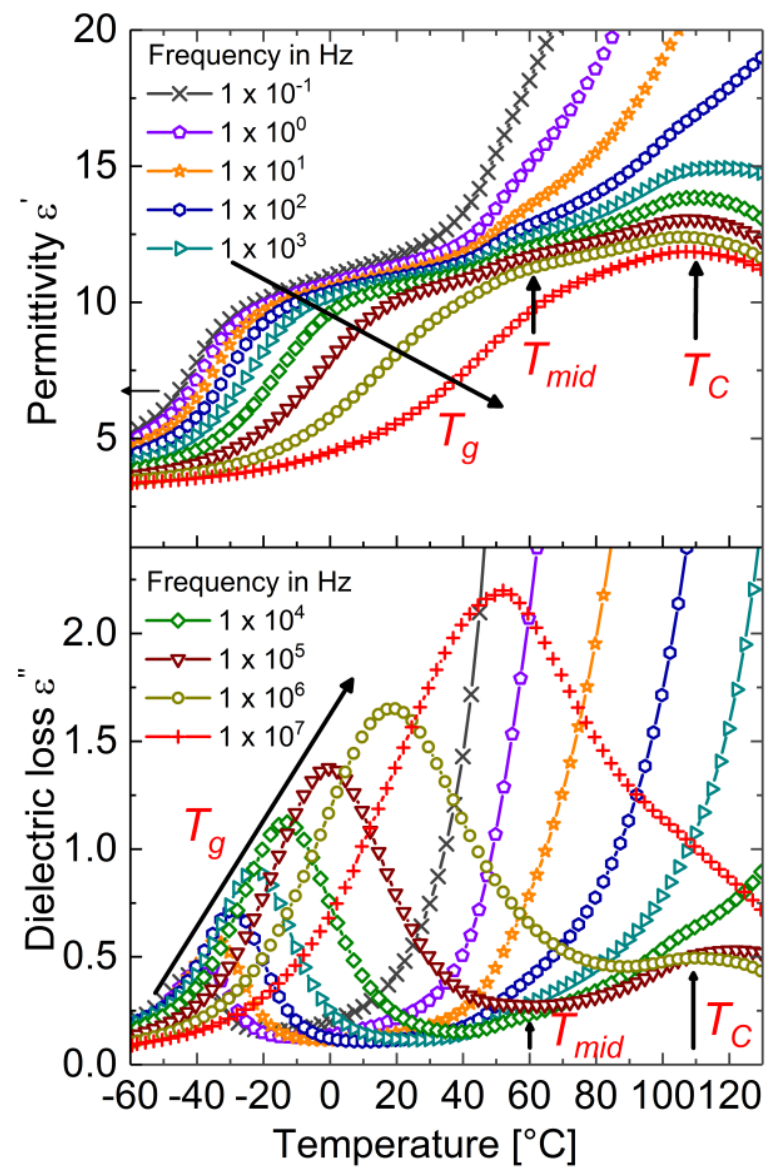

Figure 6. The permittivity and dielectric loss of a P(VDF-TFE) film stretched at $80^{\circ} \mathrm{C}$ plotted as a function of temperature at fixed frequencies from $10^{-1}$ to $10^{7} \mathrm{~Hz}$.

(Figure 4). If we look closely enough, we see the midtemperature peaks are frequency-independent in nature. So, the proposal that this could be an upper glass transition is not sufficient - or at least it does not provide the whole picture behind the mysterious transition. We have also observed similar frequency-independent peaks during DMA measurements. This has also been previously observed by Frübing et al. (University of Potsdam) in stretched films of a poly(vinylidenefluoridehexafluoropropylene) $\mathrm{P}(\mathrm{VDF}-\mathrm{HFP})$ copolymer film several years ago [31].

Next, there was another proposal. In Figure 7, we see again the DSC plot of a P(VDF-TFE) film stretched at $110^{\circ} \mathrm{C}$. During the first heating run (black curve on top), we see the $T_{\text {mid }}$ endothermic peak at around $60{ }^{\circ} \mathrm{C}$. However, when we cool the sample and heat it again, this peak is not showing-up again. So, the frequency independence together with the obviously transient nature of the endothermic peak in the second heating cycle lead to the explanation or argument that the peak could be a result of the melting of the secondary crystallites which are supposed to be formed when the sample is stored for a period of time above the glass-transition temperature of the material $[31,36]$. However, again we have a problem. Although, we do not find the peak in the second heating cycle, we see it in the 
second cooling cycle as an exothermic peak (Figure 8). Also, the exothermic peak appears in all the cooling cycles at the same temperature. As a result, this explanation also falls short of providing a complete picture.

In addition to the above two explanations, there was another hypothesis that has been put forward. It was suggested that the fluorine atoms which are in the $T G^{+} T G^{-}$chains ( $\alpha$-phase) flip from up to down or from down to $u p$ at this mid-temperature transition $\left(T G^{+} T G^{-} \rightarrow G^{-} T G^{+} T\right)[31,37,38]$. The authors called the phenomenon conformational disorder (condis) related to an up-down equilibrium occurring primarily in the crystalline

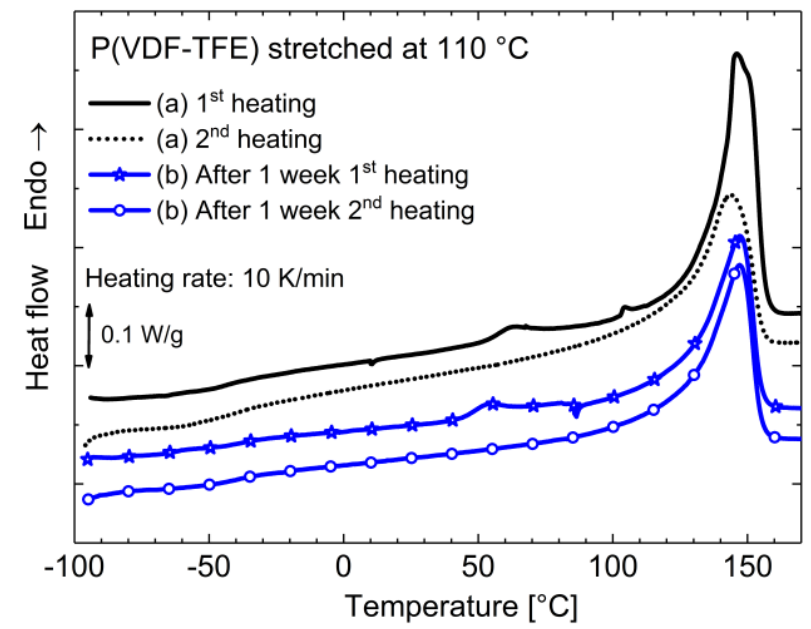

Figure 7. DSC endotherms during first and second heating cycle (black continuous and dotted curves) before and after subsequent storage at room temperature (blue curves with symbols) of a P(VDF-TFE) copolymer prestretched at $110{ }^{\circ} \mathrm{C}$. Symbols have been added to continuously recorded curves to facilitate their identification. Heating rate of $10 \mathrm{~K} / \mathrm{min}$.

regions and also in the amorphous phase of the material [31]. It was described as an irreversible relaxation. In addition, it was stated that it could normally be found only in the case of quenched or un-stretched films in which the crystallinity is not uniform [31, 38]. However, as stated before, irrespective of the processing conditions, even in the slowly cooled and the stretched samples, we definitely find this relaxation or midtemperature transition (seen in Figure 2). Again, even though this relaxation was stated to be an irreversible process, it can be actually shown that this is indeed not the case. The endothermic peak associated with the mid-temperature transition, which disappears after the first heating, actually reappears after one week -as clearly seen in the heating cycle of Figure 7 (blue curve). So, if a sufficient amount of time is available, e.g. one week, we see that the process becomes visible again. Hence, it is a reversible relaxation, and in addition, we always find the transition in the cooling exotherm. So, the third explanation also does not provide a complete overview of the process.

Another point that we have to take into account and that has so far not been considered, is the amorphous-crystalline interface (a-c). It is very interesting to note that for the same mid-temperature range where we observe the $T_{\text {mid }}$ transition, a depolarization peak was found in PVDF by Rollik et al. (University of Potsdam) already two decades ago [39]. In our previous experiments on $\mathrm{P}(\mathrm{VDF}-\mathrm{TrFE})$ copolymers and $\mathrm{P}(\mathrm{VDF}-\mathrm{TrFE}-\mathrm{CFE})$ terpolymers, exactly in the same temperature range, we have also observed similar depolarization peaks [40]. Previously, the peak was suggested to arise from the Maxwell-Wagner (MW) interface polarization, which relies on the accumulation of (compensation) charges at the a-c interface [39]. So, it becomes very obvious and important to also consider the interface effects when one wants to describe this mid-temperature transition.

Apart from the scientific interest to better understand the underlying mechanisms of this transition, its origin is also very relevant from a practical point of view because - as shown

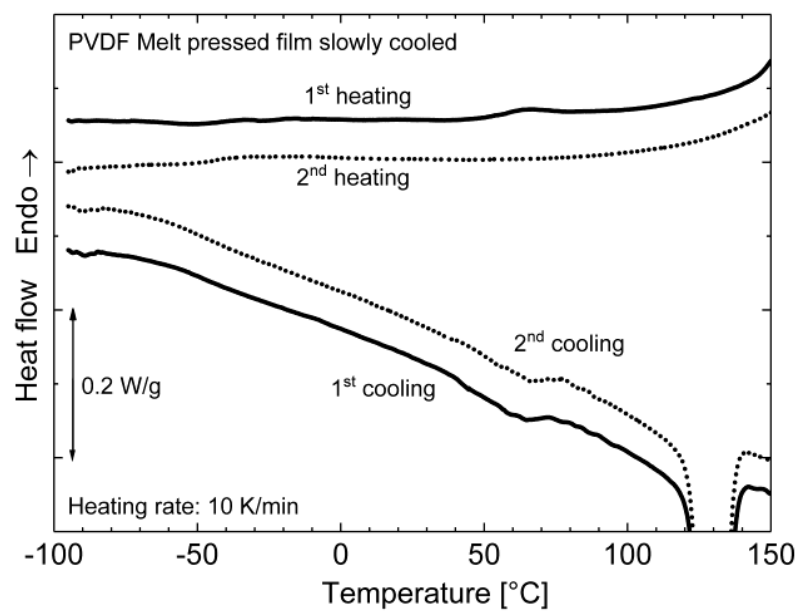

Figure 8. DSC endotherms of a melt pressed PVDF film during first and second heating and cooling cycles. Heating rate of $10 \mathrm{~K} / \mathrm{min}$.

before - mid-temperature transition affects the other properties such as aging, shrinkage, decay of piezo-, pyro-electric properties above its temperature range [41-43]. Furthermore, Teyssèdre et al. have stated that $T_{\text {mid }}$ marks the onset of orderdisorder mechanism that ultimately leads to the Ferroelectricto-Paraelectric transition (Curie transition) in ferroelectric polymers [35].

\subsection{INTERRELATED ORIGIN(S) OF THE MID- TEMPERATURE TRANSITION - HYPOTHESIS}

Now, after a review of various references in the existing literature, we can actually see that some of the mechanisms proposed to explain the mid-temperature transition overlap. Even though the various authors used different terms or different ways of explaining, there are enough indications that the transition in question has interrelated origins [44]. Furthermore, with the evidence gained from additional experiments, a new hypothesis can be formulated. Accordingly, the mid-temperature transition can be actually considered as an interrelated process where all the above-mentioned effects take place within a rather small temperature range [11]. Let us consider the amorphous-crystalline interface where we have amorphous chains pinned at both sides to crystallites. These chain segments are usually known as tie molecules [33]. In addition, we have chain loops and cilia (i.e. hair-like extensions) at the amorphous-crystalline interface [36]. When the sample is below the conventional glass transition, all these amorphous chains are frozen. Above the glass transition, the "normal", almost un-constrained chains with random conformations (i.e those found inside a more or less continuous 
amorphous phase) unfreeze. However, the constrained amorphous phase (CAP) stuck between the crystallites still remain frozen. At the mid-temperature transition or just above it, the tie molecules along with the constrained amorphous phase unfreeze-leading to an upper glass transition step. On further heating, they gain sufficient energy and also an additional degree of freedom to undergo conformational disorder. Then when we still heat the sample, the imperfect crystals that might have formed because of waiting (storage) or of quenching from the melt, result in the endothermic peak that we see in the mid-temperature range of the DSC curves. A proposed schematic of the mid-temperature range mechanisms are shown with in a previous publication [44].

With the new hypothesis, one can actually explain the individual contributions of the above-mentioned mechanisms to the mid-temperature transition for different experimental situations. For example, in case of the samples that are stretched at high temperatures, one would expect the least amount of CAP and/or tie molecules, as the molecules can be easily oriented in a soft state - facilitated by the high temperature during stretching. Here, the contribution would be expected to come
The predictions of the hypothesis are schematically visualized in Table I and can be directly applied to the DSC curves shown in Figure 2. For example, in case of the samples stretched at high temperature such as a P(VDF-TFE) film stretched at 120 ${ }^{\circ} \mathrm{C}$, we see that the mid-temperature transition looks like a relaxation. On the other hand, a P(VDF-TFE) film that was stretched at the lower temperature of $80{ }^{\circ} \mathrm{C}$ shows a small endothermic peak as predicted. Considering an annealed and slowly cooled sample, we predict the weakest relaxation. That is what we observe from the curve of a PVDF sample slowly cooled from the melt. Finally, the DSC heating curves of a non-annealed $\mathrm{P}(\mathrm{VDF}-\mathrm{TrFE})$ film, an un-stretched $\mathrm{P}(\mathrm{VDF}-\mathrm{TFE})$ sample and a quenched $\mathrm{P}(\mathrm{VDF}-\mathrm{TrFE}-\mathrm{CFE})$ film all shown in Figure 2, exhibit a strong endothermic peak as listed in Table 1.

In summary: What is the mid-temperature transition? Is it an upper glass transition? Or is it the result of secondary crystallization, as some authors have stated? Then, we also have to consider the relaxation effects due to conformational disorder and the effects of the interfaces. Is it, according to the new hypothesis, an interplay of all those effects? So, when we put all the different explanations as pieces of a puzzle together, can

Table 1. Prediction of the type of the mid-temperature transition to be seen in DSC for different heat treatments.

\begin{tabular}{|c|c|c|c|c|c|}
\hline Heat treatment & $\begin{array}{c}\text { Number of tie } \\
\text { molecules/Volume } \\
\text { fraction of CAP }\end{array}$ & $\boldsymbol{T}_{g}(\boldsymbol{U})$ & $\begin{array}{r}\text { Conformational } \\
\text { disorder (condis) }\end{array}$ & $\begin{array}{c}\text { Secondary } \\
\text { crystallization }\end{array}$ & $T_{m i d}$ expected in DSC as \\
\hline Stretched (high temperature) & Low & & & & Relaxation step \\
\hline Stretched (low temperature) & Moderate & & & & Small endothermic peak \\
\hline Annealed/ slowly cooled & Lowest & & & & Weakest relaxation step \\
\hline $\begin{array}{l}\text { Quenched/un-stretched/non- } \\
\text { annealed }\end{array}$ & High & & & & Large endothermic peak \\
\hline
\end{tabular}

mostly from conformational disorder, because it is present in all the VDF-based materials irrespective of the processing condition. So, in the end, what we would expect is a small relaxation step in DSC. When we have samples stretched at low temperatures, there is a possibility for a certain number of tie molecules/CAP to exist. Though we would have contributions from all three mechanisms in this situation, overall, due to the moderate number of tie molecules and a lower volume fraction of CAP, we would expect a smaller endothermic DSC peak. Then, in a sample that has been annealed or slowly cooled from the melt, annealing improves the crystallinity and reduces the constrained phases, and this would again lead to a low number of tie molecules. Even though there would be a contribution of condis, it will still be a minimal contribution, and the end result is that we would expect to have the weakest relaxation in the DSC plot. Finally, when we quench the sample or when it is in the unstretched state or when it is non-annealed, there are a lot of opportunities for tie molecules to develop or exist. Also, in these situations the volume fraction of CAP would be greater and we would also have a large a-c interface area. In this case, we can expect strong contributions from all three mechanisms and especially from secondary crystallization that would lead to the expectation of a strong endothermic peak in the DSC curves. the puzzle be solved? The hypothesis will be discussed in further detail mainly in Section 8 below.

\section{LOOKING AT THE DIELECTRIC DATA VIA DERIVATIVE TECHNIQUES}

If we revisit the dielectric data of a non-stretched P(VDF-TFE) copolymer film as shown in Figure 5, the peak height of the mid-temperature peak increases (frequency-independent peaks according to Figure 6). Looking at the relaxation time of these peaks, we don't see any change in the temperature dependence of the relaxation time, which is a bit contrary to what one would expect. Typically, if the amplitude of motion increases, the relaxation time is also affected to some extent. At least, within the accuracy of the data we have, we cannot see any change in the activation energy of that strong process. We see that the first process represents the dynamic glass-transition process or $\alpha$ process. The second process is very much affected by the cooling or heating or stretching history of a sample (as shown 
in Figure 2). Although we can already identify the different processes, it is better to take derivatives in order to reveal changes and their frequency dependence. In order to understand the fundamentals of the derivative techniques, the reader is referred to the work of van Turnhout and Wübbenhorst [45], [46], where a whole toolbox of derivative techniques - and the things which one can learn from them-have been summarized. Here, these techniques are applied to the dielectric data of $\mathrm{P}(\mathrm{VDF}-\mathrm{TFE})$ already shown in Figure 5, and the results are presented in Figure 9 showing two specific derivatives in $\varepsilon^{\prime}$.

The first one (upper curve) is the derivative in the frequency space $\varepsilon^{\prime \prime}{ }_{\text {deriv }}=-\frac{\pi}{2} \frac{\partial \varepsilon^{\prime}(\omega)}{\partial \ln \omega} \approx \varepsilon^{\prime \prime}$ that represents an approximation of the "conduction - free" dielectric-loss, which is particular accurate for broad peaks. In case of narrower relaxation functions, ultimately a Debye peak, the approximation that is based on a series expansion of the Kramers-Kronig transform will yield a peak being even narrower than a Debye peak. However, the shape does not

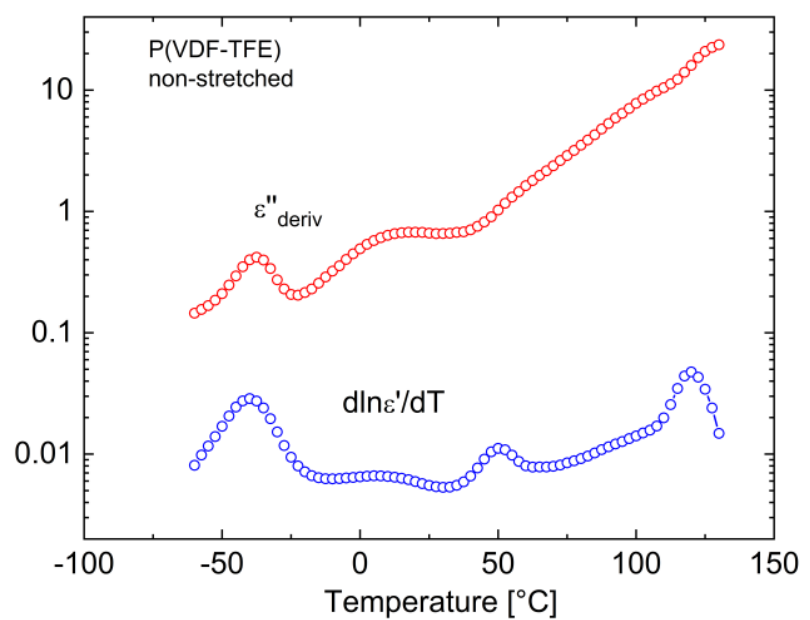

Figure 9. Isochronal representation of the "conduction-free" dielectric loss $\varepsilon^{\prime \prime}{ }_{\text {deriv }}$ (upper curve) along with the temperature coefficient of the permittivity $\partial \ln \varepsilon^{\prime}(T) / \partial T=\alpha_{\varepsilon}$ of un-stretched P(VDF-TFE) at $1 \mathrm{kHz}$.

matter here, and what we see in Figure 9 is the derivative $\varepsilon^{\prime \prime}$ deriv as function of the temperature. The first peak here is the glasstransition relaxation peak. Surprisingly, this curve shows no sign of an event around $50^{\circ} \mathrm{C}$ implying that this transition has no link with the frequency domain as expected for any thermally activated relaxation obeying time-temperature superposition. In contrast, the blue curve representing the temperature variation of $\varepsilon^{\prime}$ in the temperature space, clearly reveals a peak around $50^{\circ} \mathrm{C}$ that resembles the endothermic DSC peak. Of course, there is a different origin; by taking a derivative $\frac{1}{\varepsilon^{\prime}} \frac{\partial \varepsilon^{\prime}(T)}{\partial T}=\frac{\partial \ln \varepsilon^{\prime}(T)}{\partial T}=\alpha_{\varepsilon}$, we can obtain the temperature coefficient $\alpha_{\varepsilon}$ of the permittivity right away. In Figure 9, this quantity appears to be sensitive to the "mysterious" mid-temperature transition as well as to the melting transition as expected.

Now, let us check for the frequency dependence of the temperature coefficient of the permittivity in the temperature regime shown in Figure 10. Here, $\alpha_{\varepsilon}$ for a wide range of frequencies is plotted and the glass-transition peak shifts systematically as expected. A systematic shift is also found for the second relaxation process; here the shift is much more pronounced indicating a lower activation energy of this

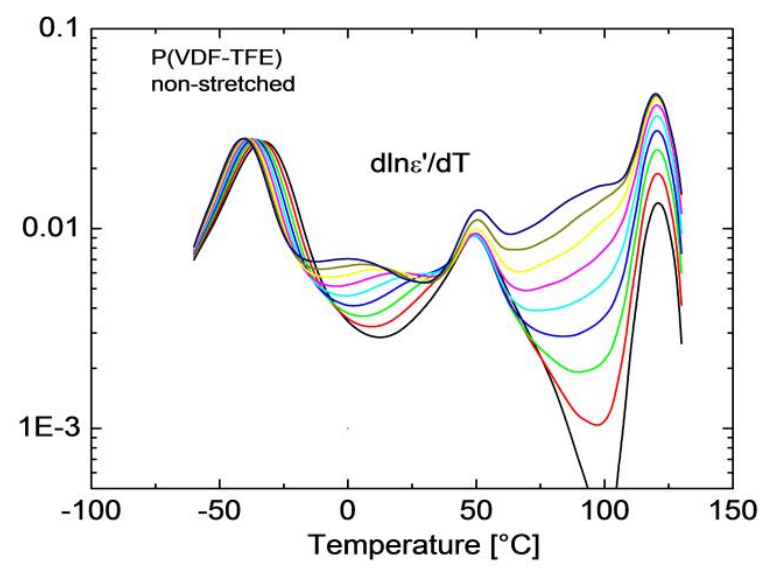

Figure 10. Temperature coefficient of the permittivity $\partial \ln \varepsilon^{\prime}(T) / \partial T=\alpha_{\varepsilon}$ of un-stretched P(VDF-TFE) at different frequencies during heating.

relaxation mode. In contrast, the peak under discussion at $50^{\circ} \mathrm{C}$, does not move at all as a function of frequency. Therefore, we can conclude that this peak is truly frequency-independent, at least in the frequency-time range we are considering (at and above $1 \mathrm{~Hz}$ ). So, it can be stated that there is a kind of structural transition that affects the density or fluctuation angle of molecular motions, which we can actually see when we analyze the second relaxation process in detail. If you fit these processes rigorously, we can show that the intermediate relaxation process shows also a step-wise gain in the relaxation strength at $50{ }^{\circ} \mathrm{C}$ - indicating that indeed the (angular) fluctuation amplitude or the number of dipoles involved in this

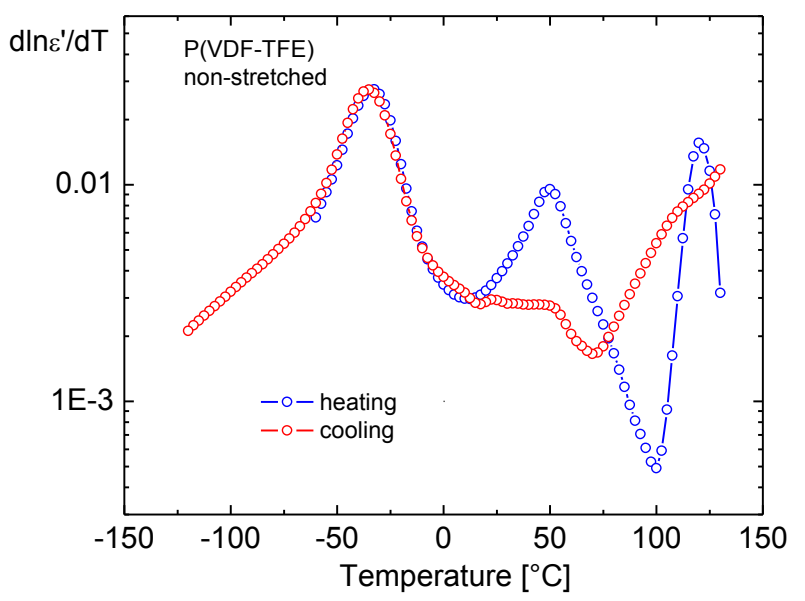

Figure 11. Temperature coefficient of the permittivity $\partial \ln ^{\prime}(T) / \partial T=$ $\alpha_{\varepsilon}$ of un-stretched P(VDF-TFE) at $1 \mathrm{kHz}$ for heating and subsequent cooling.

molecular motion is suddenly increased. One might speculate about the mechanism that facilitates this sudden gain in molecular fluctuation amplitude, however, the findings have the signature of a thermodynamic transition (of first order) that somehow affects the constrained amorphous phase. Finally, in 
Figure 11, the cooling and heating curves of the temperature depending quantity $\alpha_{\varepsilon}(T)$ of un-stretched P(VDF-TFE) are compared. The heating curve shows a very pronounced peak in that derivative, but upon cooling we also see this. It is weak, but you can still detect it by just inspecting $\varepsilon^{\prime}$ or $\varepsilon^{\prime \prime}$. Thus, the midtemperature transition as mentioned before, is also present in the cooling cycle.

\section{DOES THE MID-TEMPERATURE TRANSITION ALSO DEPEND ON THE STATE OF VDF POLYMERS?}

In Figure 12, the temperature dependence of the dielectric constant of thin films of a P(VDF-TrFE) copolymer with 75/25 mol\% of VDF/TrFE is displayed. During heating (red curve), at around $70^{\circ} \mathrm{C}$ in the dielectric loss curve, we observe the socalled mid-temperature peak. The Curie transition peak is found at a higher temperature of around $120{ }^{\circ} \mathrm{C}$; during cooling (blue curve), it appears around $80{ }^{\circ} \mathrm{C}$ in the loss plot followed by a broad peak in the range of the mid-temperature transition. These results are from $\mathrm{P}(\mathrm{VDF}-\mathrm{TrFE})$ samples in the form of thin films.

Now, let us discuss the DSC measurements on P(VDF-TrFE) copolymer with different molar ratios of VDF/TrFE in their powder form which are shown in Figure 13. The samples were heated from room temperature. In the Figure, we do not see any clear exothermic peaks in the mid-temperature region for all three compositions of VDF/TrFE. It seems to be that the midtemperature transition is absent in the co-polymer in its powder form. May be the phenomenon is only observed in films? The processing should have some effect on this transition. There is also the possibility of residual solvent in the film. May-be somethings change during or after film fabrication. This could be checked using FTIR. The small amount of solvent that may be present may cause the difference in the amorphous phase locally. May be in this case, if we increase the temperature of the copolymer film, the solvent may evaporate and we may have only the typical amorphous regions (without the midtemperature transition)? So, this should be a possible reason why DSC measurements of the copolymer powder do not show any peak around 50 to $70^{\circ} \mathrm{C}$.

T.R.V mentioned that in the case of polymers in their powder state, it is not clear to what extent the conditions favoring the formation of secondary crystals exist, as usually their formation requires a thermal step during processing where the polymer is heated to a high temperature near or above the melting point $[45,48]$. Also, for the conformational disorder (condis) to take place, we need a stable $\alpha$-phase. In addition, the role of the interaction between the amorphous and crystalline phases at interfaces must be considered. So, when the sample is in powder form, it is also not clear to which extend this $a-c$ interaction is feasible. So, maybe not the solvent, but the a-c interaction and the existence of loops, tie-molecules and cilia might play a decisive role?

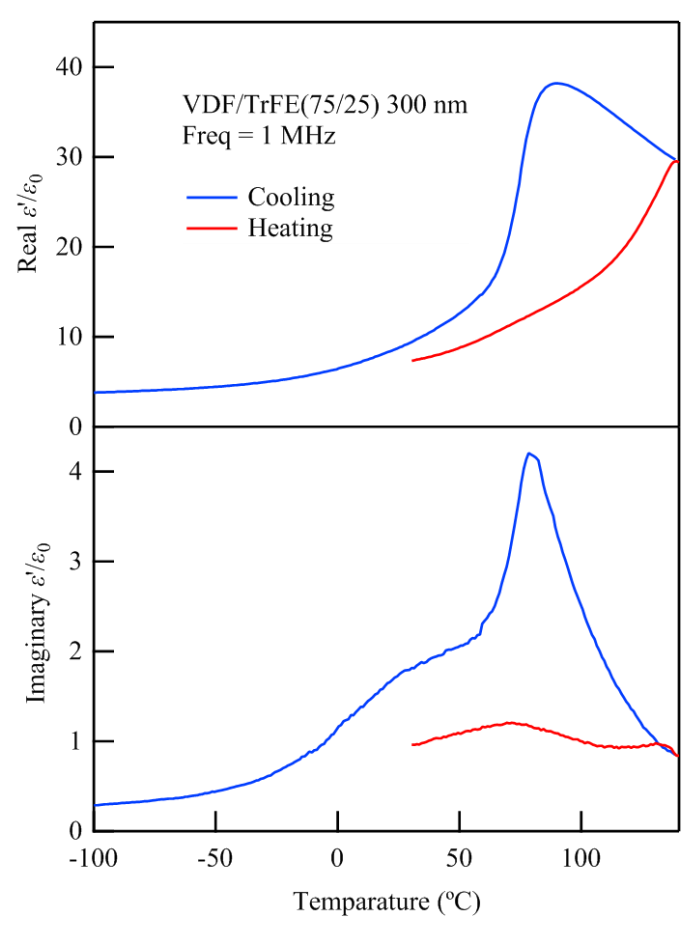

Figure 12. The permittivity and dielectric loss of an unstretched P(VDF$\mathrm{TrFE}$ ) film as a function of temperature at $1 \mathrm{MHz}$ during heating from room temperature and subsequent cooling.

\section{POSSIBLE EFFECTS OF ELECTRIC POLING ON THE MID-TEMPERATURE TRANSITION}

The dielectric relaxation and polarization phenomena in polymers are always more complicated than in the case of ceramics or other materials. Now, from the above hypothesis, we know it is further more complicated. If we consider a $\mathrm{P}(\mathrm{VDF}-\mathrm{TrFE})$ copolymer, it is a very complex material because we have different phases; A crystalline phase, an amorphous phase, and the interfaces between them. Even the respective crystalline and amorphous phases are not all the same, because they can change not just instantaneously (e.g. during quenching), but also with time (secondary crystallization). This can already be seen from poling tests on them. The results show also some kind of similar behavior to that of dielectric measurements. For example, when we measure the dielectric spectrum before and after poling, we can sometimes see big differences. This means not only with thermal treatment, but also with poling, i.e. under an electrical field, the properties can change a lot. Still, it is not clear what kind of changes are happening. It was pointed out that during poling, charges can be injected and may be trapped at the amorphous-crystalline interfaces and may be responsible for the Maxwell-Wagner polarization, which-as stated before-has already been observed in Thermally Stimulated Discharge-Currents (TSDC) results on the homopolymer and terpolymer, respectively [39, 40]. 
Thus, during poling, many things are happening in the material. The molecules are re-arranged. Then, the crystallinity also increases to some extent. In addition, the polarization depends very strongly on the poling history, which means that we have to cycle the electric field many times and that something is also happening during the poling process. We can see this in Figure 14 where the remanent polarization $\left(P_{r}\right)$ keeps

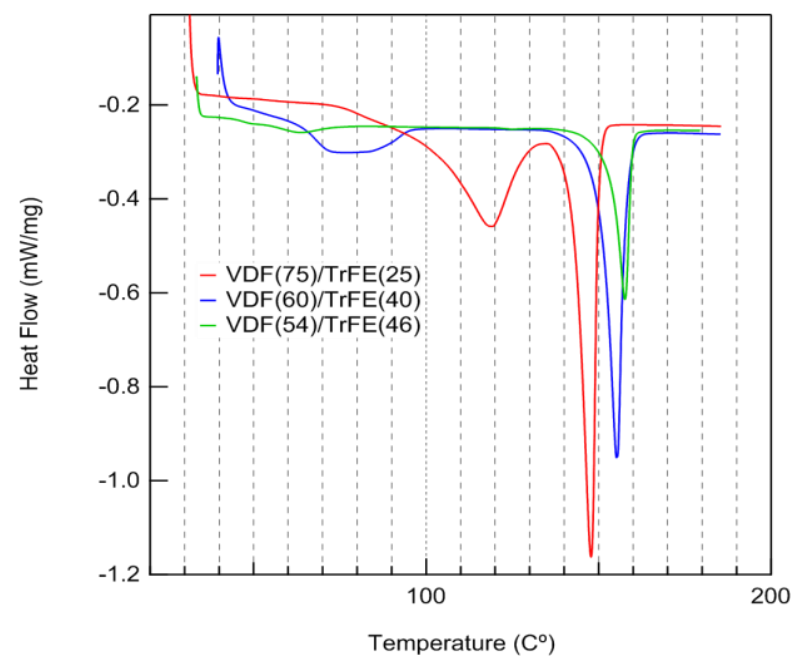

Figure 13. DSC endotherms of different compositions of $\mathrm{P}(\mathrm{VDF}-\mathrm{TrFE})$ in their powder form.

growing with the number of poling cycles in a $\mathrm{P}(\mathrm{VDF}-\mathrm{TrFE})$ sample with $75 / 25 \mathrm{~mol} \%$ of VDF/TrFE. What we observe is that when you cycle the poling field only once, $P_{r}$ is relatively low. If we apply more cycles, $P_{r}$ keeps increasing and saturates after 10 to 20 cycles. That means, in order to achieve a stable state or a relative equilibrium, it is very important that we cycle the sample many, many times. In principle, it is similar to the situation where we have to heat or subject the sample to a thermal treatment for a period of time so that the material becomes more stable. The above explanation of the midtemperature is very interesting with respect to the amorphouscrystalline interfaces, and this probably can be checked using samples with different crystallinity. From the literature, it is possible to have a copolymer with up to $100 \%$ crystallinity. So, we can change the crystallinity and see how the behavior changes. Maybe this will give us a clearer clue on what is exactly going on in the material.

With respect to the electric polarization behavior of P(VDFTrFE) copolymers, T.R.V. suggested to look at experiments on commercial P(VDF-TrFE) films poled by means of the socalled "Bauer cycling method" as suggested by François Bauer et al. [48, 49]. Commercial P(VDF-TrFE) films were subjected to about 100 poling cycles and changes in $P_{r}$ were observed. It was also noticed that $P_{r}$ changes with the $\mathrm{VDF} / \mathrm{TrFE}$ composition [50]. For example, 75/25 mol\% of VDF/TrFE was the optimal composition where a relatively higher $P_{r}$ was achieved at a relatively lower number of poling cycles. However, in the case of films with quite a similar mol\% composition of 72/28, $P_{r}$ increases to twice its original value at 20 cycles when the films were subjected to a higher number (100) of poling cycles. When there is a change in composition, there is also a change in the fraction of $\beta$-phase or $\alpha$-phase andas previously mentioned above-the crystallinity also changes. Sometimes, even 100 cycles are not enough to achieve a saturation in $P_{r}$, as further growth in $P_{r}$ could be observed [50]. By means of Piezoelectrically generated Pressure Steps (PPSs), the distribution of the polarization in the thickness direction can be probed. PPS scans on the P(VDF-TrFE) samples showed a non-uniform polarization profile even after hundreds of cycles, which is not expected in the case of a saturation in $\operatorname{Pr}_{r}$ [50]. The other problem with further increase in the number of poling cycle is the risk of injecting charges into the sample, thus provoking an early electrical breakdown in the sample. This risk prevented a further increase in the number of poling cycles. Nevertheless, as mentioned before, measuring the electrical hysteresis may also provide information about the origins behind the mid-temperature transition, as the phase content of the samples could be changed by means of electric poling and the possible effects on the mid-temperature transition could be further investigated.

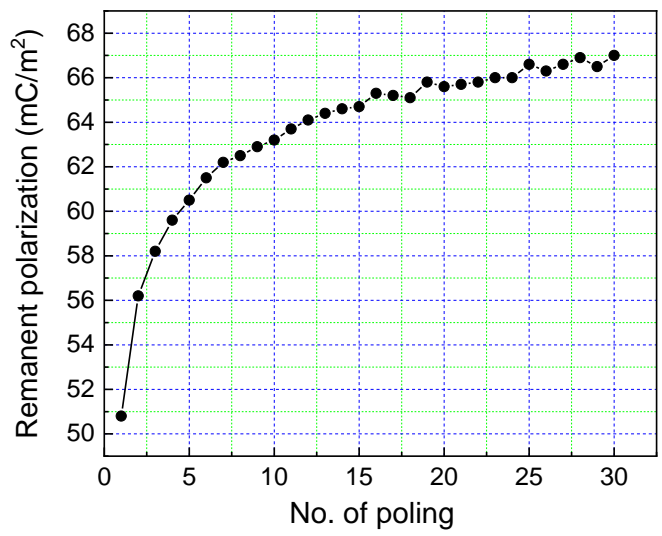

Figure 14. Remanent polarization as a function of the number of poling cycles in a P(VDF-TrFE) $(75 / 25 \mathrm{~mol} \%$ of VDF/TrFE) film. Poling was done by applying two cycles of a bipolar triangular voltage sweep with an amplitude $\mathrm{V}_{\mathrm{am}}$ of $1.8 \mathrm{kV}$ (i.e. with an $E_{a m}$ of $100 \mathrm{MV} / \mathrm{m}$ in the sample).

\section{MID-TEMPERATURE TRANSITION IN ULTRA THIN FILMS SEEN THROUGH NON-LINEAR DIELECTRIC SPECTROSCOPY}

From the series expansion of dielectric displacement $D$ expressed as a function of the applied electric field $E$ we have:

$$
D=P_{S}+\varepsilon_{0} \varepsilon_{1} E+\varepsilon_{0} \varepsilon_{2} E^{2}+\ldots
$$

where $\varepsilon_{1}$ (commonly expressed as $\varepsilon$ ) is the $0^{\text {th }}$ order non-linear permittivity which is normally measured using DRS. In addition to it, we have the $1^{\text {st }}$ order non-linear permittivity $\varepsilon_{2}$ expressed in Equation (1). To measure the non-linear permittivities, a sinusoidal electric field which is far below the coercive field of the sample is applied and the corresponding 
spectra is obtained by the Fourier Transform of the output current density $j(t)$ as shown in Equation (2) below:

$$
j(t)=\frac{\mathrm{d} D(t)}{\mathrm{d} t}=\sum_{l=0}^{\infty}\left(j^{\prime}{ }_{l} \cos l \omega_{0} t+j^{\prime \prime}{ }_{l} \sin l \omega_{0} t\right)
$$

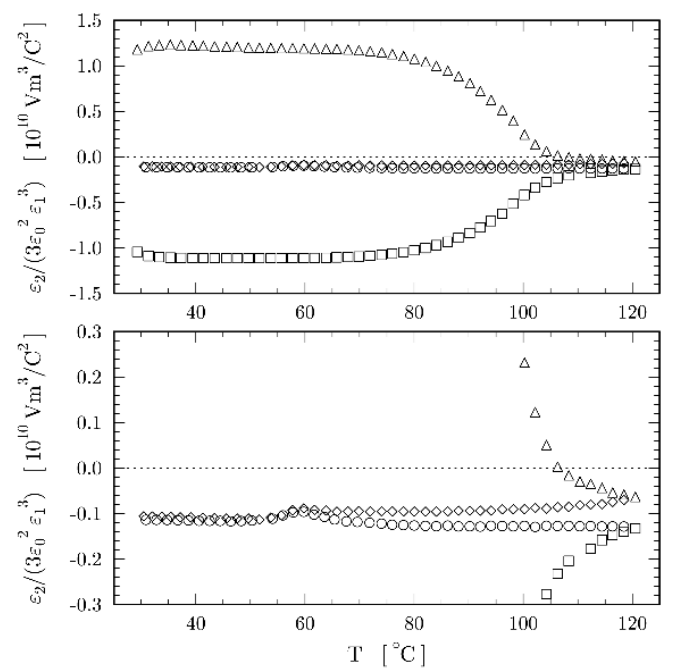

Figure 15. Quotient $\varepsilon_{2} /\left(3 \varepsilon_{0}^{2} \varepsilon_{1}^{3}\right)$ which is proportional to the remanent polarization $P_{r}$ as a function of temperature T for a $64 \mathrm{~nm}$ thick annealed $\mathrm{P}(\mathrm{VDF}-\mathrm{TrFE})$ copolymer film of molar ratio $70 / 30 \mathrm{~mol} \%$. Film polarized at room temperature by a positive voltage at the top electrode before the heating $(\Delta)$ and cooling $(\diamond)$ cycle, and poled by a negative voltage at the top electrode at room temperature before the heating $(\square)$ and cooling $(\circ)$ cycle. In the lower diagram the vertical axis is spread. Data from [51].

Finally, the non-linear permittivities $\varepsilon_{n}$ can be calculated from Equation (3), in the case the excitation amplitude is chosen sufficiently small so that the coefficients $j^{\prime \prime}{ }_{n}$ decrease strongly with increasing order of $n$.

$$
\varepsilon_{0} \varepsilon_{n} \approx \frac{-1}{\omega_{0}} \frac{2^{n-1}}{n E_{\sim}^{n}} j_{n}^{\prime \prime}
$$

While the real part $\left(\varepsilon_{n}^{\prime}\right)$ of the complex non-linear permittivities are referred to in Equations (1) and (3), the imaginary part $\left(\varepsilon_{n}^{\prime \prime}\right)$ is calculated from the non-vanishing $j_{n}^{\prime}$ components using an analogous equation to Equation (3) where $j_{n}^{\prime \prime}$ is replaced by $j_{n}^{\prime}$.

In Figure 15, the non-linear polarization in $\mathrm{P}(\mathrm{VDF}-\mathrm{TrFE})$ copolymer thin films (about $65 \mathrm{~nm}$ thick) is plotted as a function of temperature. After the preparation, electrodes are deposited on top of the sample, and the sample is annealed at about 120 or $130{ }^{\circ} \mathrm{C}$ (close to its melting point) in order to improve the crystallinity, and it is then polarized in the positive direction at room temperature. On such a sample, the polarization is recorded versus temperature by measuring dielectric non-

linearity $\varepsilon_{2}$ and linear permittivity $\varepsilon_{1}$ where the ratio $\varepsilon_{2} /\left(3 \varepsilon_{0}^{2} \varepsilon_{1}^{3}\right)$ is proportional to the remanent polarization in the sample. In Figure 15 (top), when the temperature is increased, we see that the polarization curve remains almost the same for a large temperature range, and at around $100{ }^{\circ} \mathrm{C}$, the film is depolarized. This curve is not very surprising, but when we cool the sample down, the polarization does not reduce to zero instead we get a small, but constant value. In the next step, the sample is polarized in the negative direction at room temperature and the experiment is repeated. On heating, the polarization goes down, but - again - it does not reach zero on cooling down. So, there is a remanent polarization that cannot be erased by heating the sample up to $120^{\circ} \mathrm{C}$. Now, in the bottom diagram of the same Figure, which is just the magnification of the diagram on the top, we see that there is a structure at $60{ }^{\circ} \mathrm{C}$ - just at the same temperature range where T.R.V. has observed characteristic structures again and again (cf. the experimental results and the relevant literature mentioned above). So, if it is the same mid-temperature transition, nonlinear spectroscopy indicates that it is somehow related to the electric polarization.

Now the question is: What is this polarization? To get more information, experiments like the one shown in Figure 16 were performed. The films were deposited by spin-coating again and then polarized before annealing. It is possible that the

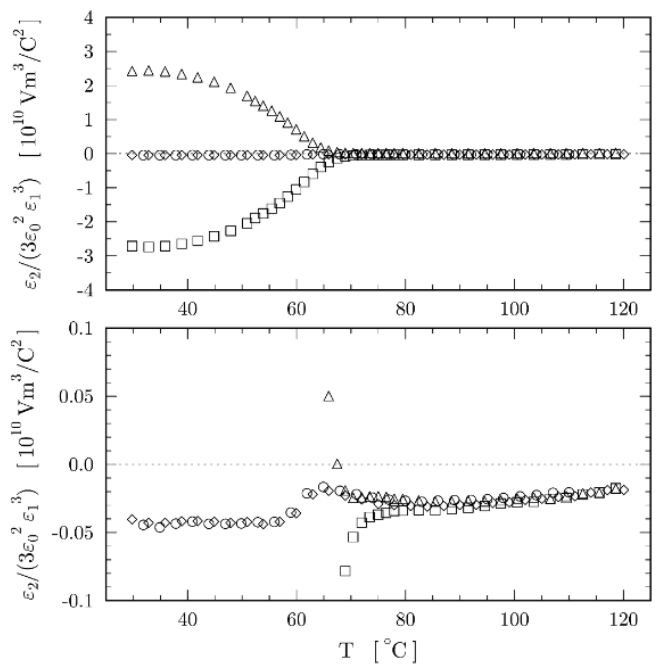

Figure 16. Quotient $\varepsilon 2 /(3 \varepsilon 02 \varepsilon 13)$ which is proportional to the remanent polarization $\mathrm{Pr}$ as a function of temperature $\mathrm{T}$ for a $2 \mu \mathrm{m}$ thick annealed VDF-TrFE copolymer film of molar ratio $56 / 44 \mathrm{~mol} \%$. The film had been polarized in positive direction before the first annealing. Film polarized at room temperature by a positive voltage at the top electrode before the heating $(\Delta)$ and cooling $(\diamond)$ cycle, and poled by a negative voltage at the top electrode at room temperature before the heating $(\square)$ and cooling $(\circ)$ cycle. In the lower diagram the vertical axis is spread. Data from [52].

crystallinity is a bit smaller, but we can see the hysteresis curve [16] and we can polarize these un-annealed films either in the positive or the negative direction and then repeat the same procedure. Then, we see an additional polarization which depends on the first polarization direction of the un-annealed film. That means if we polarize an un-annealed film in the positive direction and anneal it, cool it down, heat it, polarize it in any direction, and heat it above the Curie transition to 120 ${ }^{\circ} \mathrm{C}$, there is still a remanent polarization that cannot be erased any more. So, the films have a memory of the first polarization direction in the un-annealed state, and we can only remove this polarization by dissolving the film. So, what may happen is that during annealing the crystallinity increases. That means the crystallites grow and the explanation which may be a bit hypothetical, but may fit is: When the crystallites grow and are polar after being polarized, the bending of the entangled polymer chains that come out of the surface increases. Thus, their polarization will be no longer switchable. The explanation which we have suggested is that it happens at the surfaces of the 
crystallites and it is the polarization at the crystalline surface or the interface with the amorphous phase. So, our results fit very well to the explanation of Maxwell-Wagner polarization as observed by Denis Rollik et al. [39] and by Thulasinath Raman Venkatesan et al. [40].

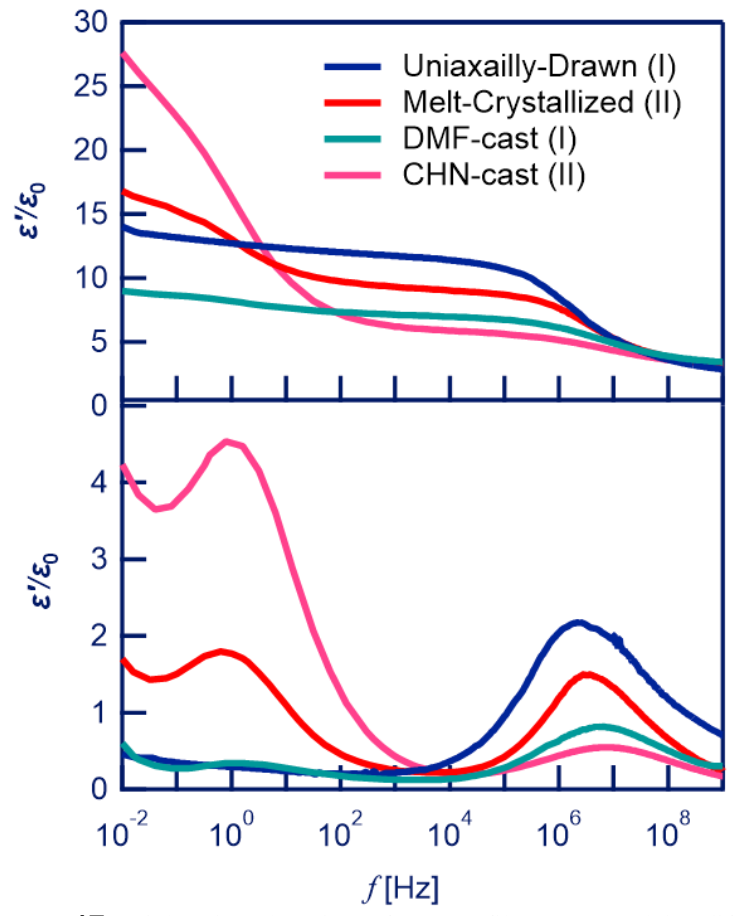

Figure 17. Dielectric relaxation of PVDF films prepared by different methods measured at $30^{\circ} \mathrm{C}$. The crystalline structure of the various films are included (in brackets) along with the respective film preparation technique. Uniaxially drawn and dimethyl formamide (DMF)-cast films consists of form I or $\beta$-phase crystals, whereas melt-crystallized (MC) and cyclohexanone ( $\mathrm{CHN}$ ) cast sample consists of primarily form II or $\alpha$-phase crystals.

\section{BROADBAND DIELECTRIC SPECTRO- SCOPY TO VIEW ALL DIELECTRIC RELAXATIONS IN VDF-BASED POLYMERS}

T.F. (Takeo Furukawa) started to explain his point of view by going to the basics of dielectric relaxation in PVDF. If we look at his data (Figure 17), we can see the dipolar relaxation of PVDF films prepared by different techniques and measured at $30{ }^{\circ} \mathrm{C}$, over a very broad frequency range from $1 \mathrm{GHz}$ all the way down to $0.01 \mathrm{~Hz}$. We can see the big relaxation loss peaks at low frequencies for form II PVDF on the left of the figure. This is a result of semi-crystalline motion. Normally, segmental motions seen on the right side of the Figure do not change shape, but the peak position will change with temperature, but this is crystalline or semi-crystalline motion, which is seen on the left. This is the whole picture in this kind of measurement, but we have to go to the $\mathrm{GHz}$ range where relaxation is seen at about $10 \mathrm{MHz}$. In Figure 18, the dielectric spectra of form II PVDF is shown. The dielectric response have been recorded in the frequency range from $1 \mathrm{mHz}$ to $10 \mathrm{GHz}$. In this broad frequency range, we can observe all molecular motions. The sample film is fully $\alpha$-phase PVDF prepared from cyclohexanone (CHN) solution [53, 54]. When we look very carefully, we observe two relaxations. As already stated, the sample is purely $\alpha$-phase PVDF. In this phase, $T G^{+} T G^{-}$chains are perpendicular to the film surface along the thickness direction. Therefore, we observe very strong $\alpha$ and crystalline relaxations. Looking at Figure 18 again, we see that the maximum dielectric strength of the semi-crystalline motions is 30 (permittivity $\varepsilon^{\prime}$ ). This is transitional motion [55] associated with the crystalline regions similar to that observed in Figure

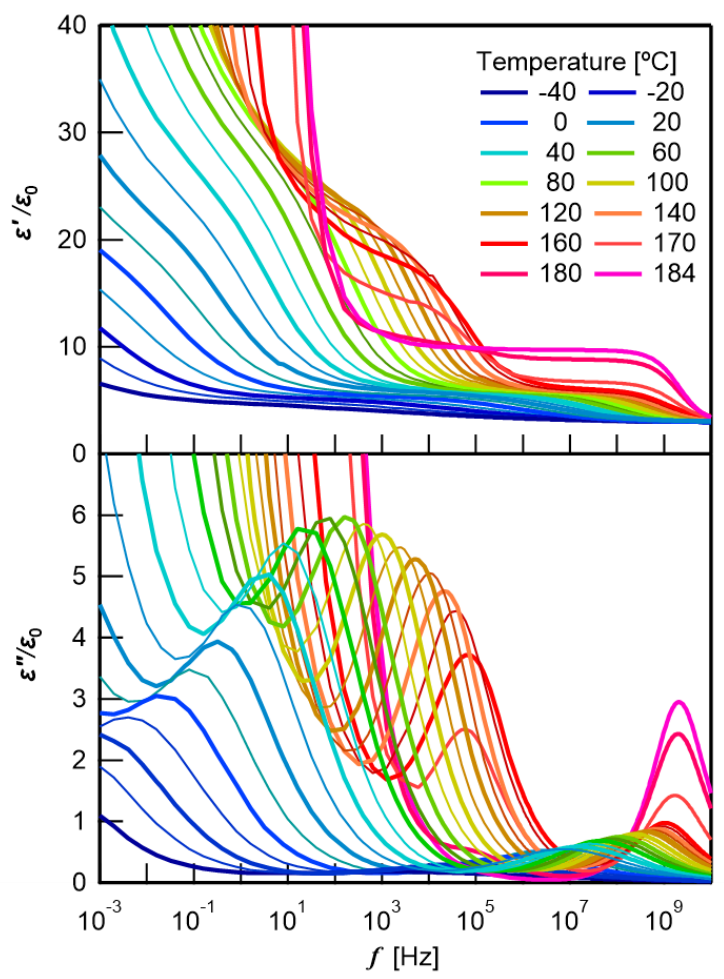

Figure 18. Dielectric spectrum of a PVDF film cast from cyclohexanone showing fully $\alpha$-phase. The real (permittivity, $\varepsilon^{\prime}$ ) and imaginary (dielectric loss, $\varepsilon^{\prime \prime}$ ) parts of the complex permittivity are plotted as a function of frequency at different temperatures.

17. Here, we also observe a small relaxation at the highfrequency end of the spectrum. It is almost symmetrical. These are so-called local motions ( $\gamma$ relaxation) taking place in the $\alpha$ phase crystalline regions that undergo both slow transitional motion and also fast localized motions ( $\alpha$ and $\gamma$ relaxations, respectively). For example, around $40^{\circ} \mathrm{C}$, the samples undergo both the $\alpha$ relaxation and localized motions. Actually, these two types of motion are co-existing, and the interesting thing here is the crystalline motion. As we increase the temperature, which is around $180{ }^{\circ} \mathrm{C}$, the transitional motions ( $\alpha$ relaxation) disappear which is because of melting and we see that the other relaxation ( $\gamma$ relaxation) goes up. So, this means that the melt regions also allow relaxation to take place. In the molten phase, the former crystalline regions and some of the local motions combine. To sum up, these are the various molecular motions in the molten phase in PVDF in the region around 1 to $2 \mathrm{GHz}$. The dielectric relaxation map of normal melt-crystallized (MC) PVDF that is primarily also in the $\alpha$-phase is shown in Figure 19 . We see that the dielectric strength is not so high - only 15 or so (permittivity $\varepsilon^{\prime}$ ). For the other sample (CHN-cast PVDF), 
it was 30. The increment is much lower in the MC-PVDF sample because the $\alpha$-phase molecular chains are randomly oriented and the transitional relaxation occurs mostly perpendicular to the chain direction. Though the strength is not the same, the frequency behavior is identical to the CHN-cast PVDF film. Here, the most important thing is that the relaxation seen in the high-frequency range from $100 \mathrm{kHz}$ to $1 \mathrm{GHz}$ has two components. Normally, most people say this relaxation is the $\beta$ relaxation (glass transition), but there are always two processes here. Below the melting temperature, we see the $\beta$ relaxations and above the melting point, we have our localized motions. We also notice that the shape of the peaks changes here so much. The best thing to do is to measure the whole available frequency range and make an analysis. The spectrum was analyzed with three separate Cole-Cole functions, and all features could be reproduced clearly. It is obvious that there are in total three processes taking place in the PVDF sample over the whole frequency range and their peaks either shift or remain constant with a change in temperature, as shown in Figures 18 and 19, and with different relaxation times.

In Figure 20, the relaxation times of the various processes in $\alpha$-phase PVDF film prepared with different methods are shown. At the bottom of the figure, we see the so-called localized motions ( $\gamma$ relaxation) in CHN-cast PVDF. Then the $\beta$ process shows Vogel-Fulcher-Tammann relaxation behavior as observed in a MC-PVDF sample. Finally, we observe the relaxation time of the $\alpha$-relaxation in both $\mathrm{CHN}$ - and $\mathrm{MC}$ PVDF. So, very clearly we see that there are three processes, but we have to cover a very broad range of frequencies to see all the processes. Melting and ferroelectric-to-paraelectric transition dynamics which are also taking place in $\beta$-PVDF films can be clearly observed by making a frequency-dependent analysis. Though many people do temperature-dependent studies at fixed frequencies, it is better to do a frequency-related analysis. Now, coming to P(VDF-TrFE), we know that the copolymer shows also a $\beta$ relaxation associated with the glass transition of the material [56]. Although an $\alpha$-process is often missing, it still occurs commonly along with $\beta$ and $\gamma$ processes in other VDF-based polymers. The segmental motions and the local motions coexist, especially near or at room temperature, and they appear at the same frequency ( $\beta$ and $\gamma$ relaxations, respectively). Therefore, we cannot distinguish between them. Only at very low temperatures (below $-40{ }^{\circ} \mathrm{C}$ ), we can distinguish them and observe the shift in peaks. These motions go all the way to the molten phase. Finally, it can be said that the processes (localized motions) observed in the molten phase and at low temperatures are essentially the same.

\section{SUMMARY OF THE DISCUSSION}

In the following, the above hypothesis and the different viewpoints advanced by the co-authors are being discussed. As there are still open questions, the discussion does not always lead to a unanimous conclusion. T.R.V. (Thulasinath Raman Venkatesan) pointed out that the concept of constrained amorphous regions which he refers to deals with the amorphous regions that are embedded between crystallites, along with the respective interfaces that do not undergo the conventional glass transition, as their chain segments are constrained on all sides by crystalline regions and thus do not have sufficient degrees of freedom to move. However, when the imperfect crystals, and especially the secondary crystals, eventually melt, they attain the necessary degrees of freedom to move. That is why the phenomenon is sometimes referred to as upper glass transition $[40,58,59]$. To summarize, the conventional $T_{g}$ is observed in the normal amorphous phase and the upper $T_{g}$ occurs when there are constrained amorphous regions with tie molecules and larger amorphous-crystalline interfacial areas.

Michael Wübbenhorst (M.W.) added that structure formation definitely takes places and includes freezing into a certain conformational order, which in a polar phase is linked to the polar order that is frozen in. Once the crystal structure is more or less fixed, as the highest possible degree of crystallinity is reached (which may be enhanced by repeated switching that

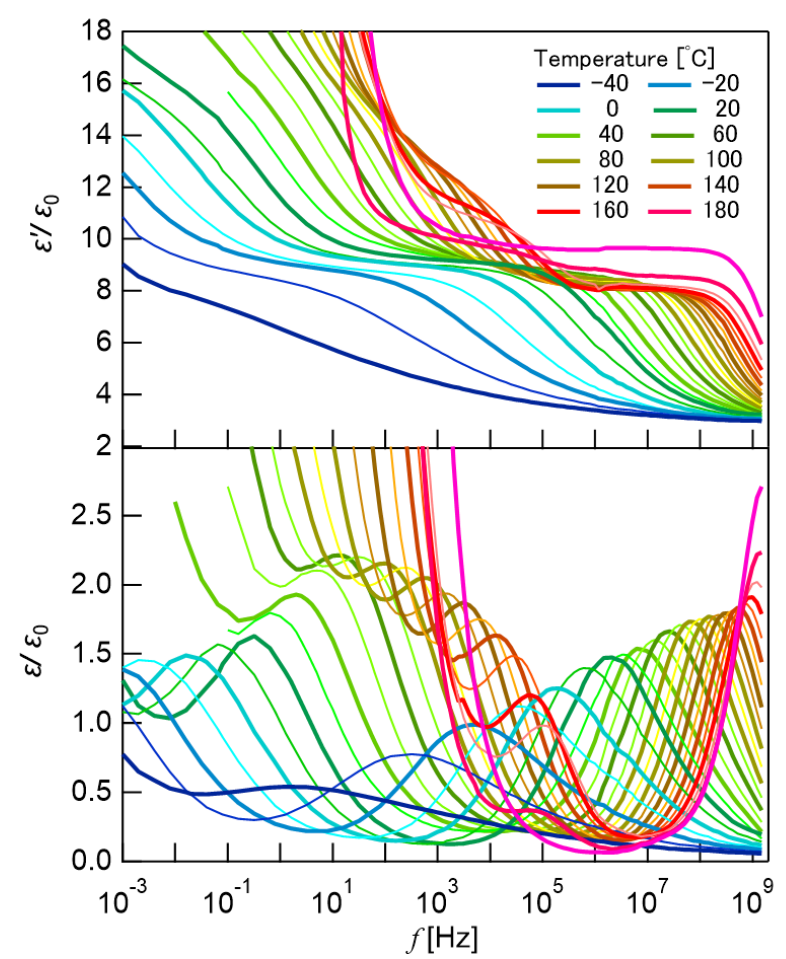

Figure 19. Dielectric spectrum of a melt-crystallized PVDF film showing fully $\alpha$-phase. The real (permittivity, $\varepsilon^{\prime}$ ) and imaginary (dielectric loss, $\varepsilon^{\prime \prime}$ ) parts of the complex permittivity are plotted as a function of frequency at different temperatures.

further improves crystalline quality), some conformations become immobilized. Electric-field cycling and annealing may also lead to fatigue after many, many cycles, and defects can be finally trapped so that the switchability decreases over time. Thermal annealing can partially reset the system, and when the sample melts, the whole thermal history is more or less erased. There might be another explanation for thin films where the specific difference is adsorption of polymer chains at the substrate surface. Interfaces are never identical, as their configurations vary due to the way in which the films are prepared. If we start with spin-coating and expose the film to high temperatures we create an absorbed layer that can never be removed. Even dissolving the polymer film is possible only down to the first, physiosorbed polymeric layer on the substrate. 
M.W. further stated that in systems involving PVDF this is what one would expect. So, it could explain why certain interfacial configurations are simply adsorbed and you will never change them and you even get surface-induced polarity which stabilizes eventually the polarization direction of the adjacent crystals. Still, the question is why we have this mysterious temperature. The idea of secondary crystals is just a semantic question. From other polymers we know that there exist other ordered phases, other ordered tiny, nanometer-sized structures that have high orientational order, eventually even polarity or not - this depends. They may even have different crystal structure, or even if not a full crystal structure, they might have just a high orientational order like a nematic structure; and these objects, as long as they have a certain size, can have a transition temperature where the orientation order is lost, something like a nematic-isotropic transition. Then it would not be so much size-dependent. M.W. went on to explain that upon cooling or other processing, as long as there are enough available mobile chains present, they form just secondary ordered structures, and he would not call them crystals. This is something one can eventually study in more detail. This is his (M.W.'s) revised view of the mid-temperature transition phenomenon, which would bring a thermodynamic aspect to it. While we have more or less always the same characteristic temperature, whatever this means (structural transition or relaxation-like) and how strong this phenomenon is, it depends on that particular structure in the material.

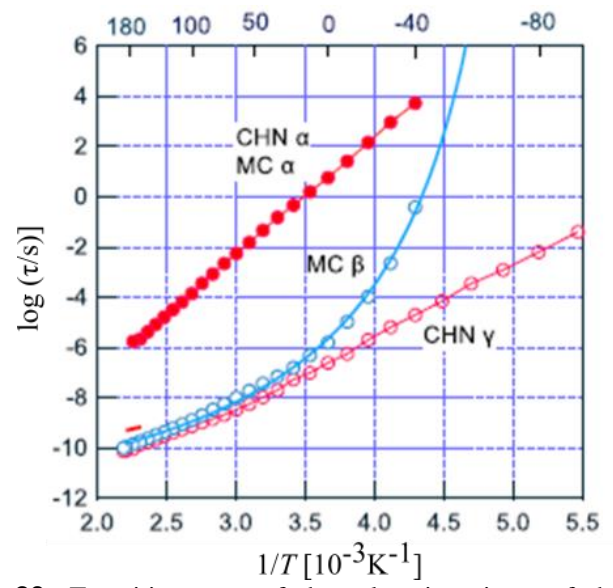

Figure 20. Transition map of the relaxation times of the different processes in cyclohexanone-cast and melt-crystallized PVDF films.

B.P. (Bernd Ploss) argued that he was not sure if the localized motions that T.F. spoke about are the same phenomenon as the mid-temperature transition. In his opinion, these are two different aspects and measuring over a wide frequency range definitely helps a lot in the case of polymers in order to observe all kinds of motion, but the peaks (related to the midtemperature transition) that M.W. discussed are much weaker than the others. M.W. has applied the differential analysis to obtain more information and his result was that - at least over a limited frequency range (5 decades from $10 \mathrm{~Hz}$ to $1 \mathrm{MHz}$ ) there was no frequency dependence for the mid-temperature process. B.P. asked how the localized motions and the frequency-independent mid-temperature transition can be brought together?
T.F. replied that one has to go to very low frequencies $(<10$ $\mathrm{mHz}$ ). At these low frequencies, it takes about 1 day to make a data point. He mentioned that his group had used four sets of equipment to obtain the complete results. Following this, B.P. expressed his opinion that though it is great to have over 12 decades in the frequency range, but that 6 decades already take time, and M.W. added that one could in principle measure more than 5 decades if required. However, both felt that in the present case, it is not necessary to use a wide range of frequencies, as the mid-temperature transition is already observed in the limited frequency range between $10 \mathrm{~Hz}$ and $1 \mathrm{MHz}$. Next, there was an argument [59] that many authors consider PVDF as a perfect structure (without defects), but indeed this is not the case because when you prepare it by free radical polymerization, you also introduce in the material about $6 \%$ defects and may-be these defects in the material are responsible for the mid-temperature transition. It was also explained that these are regional defects in the polymer. Normally, one finds head-tail (H-T) addition in PVDF, but head-head $(\mathrm{H}-\mathrm{H})$ and tail-tail (T-T) addition of the monomer ("defects") are also very common. It was argued that the defects are often not considered especially by physicists. Normally, the density of the defects is around $6 \%$, and to exactly know the percentage of defects, one has to do an NMR analysis of the polymer.

B.P. felt that the defects are not responsible for the midtemperature transition. He replied that if it really has an effect, then there will be only a local change in density of the material. T.R.V. commented that Sylvie Tencé-Girault's research group at the Arkema Centre de recherche de Serquigny (CERDATO) has done experiments in this direction and published results about the $\mathrm{H}-\mathrm{H}$ and $\mathrm{T}-\mathrm{T}$ defects [23]. They report that when you subject the material to a repeated thermal history (for example, heat to a temperature near the Curie transition and then cool back, and again heat to a slightly higher temperature and cool again, which is referred to as consecutive annealing), the $\mathrm{H}-\mathrm{H}$ and T-T defects which may be part of the structure of the primary crystals can be eliminated from crystals. After the thermal treatment, they had done small-angle X-ray diffraction (SAXD) measurements and other studies to show that the defects, which they refer to as irreversible defects, are thrown out of the ferroelectric crystals to form a defective ferroelectric phase. They describe it to be similar to the CL, the LTD or F $\beta 2$ phases already described in the literature [61, 62].

T.R.V. acknowledged that indeed these defects are always present in the material whatever you do. Similar to a crosslinking system, it is a permanent effect which cannot be removed from the material, but they can be gradually removed from the crystals and once they are removed from the ferroelectric crystals, the reports say that they cannot be reintroduced into the crystal system [60]. It does not want to go back into the crystal structure. This is because of the thermodynamic behavior of the crystal, which wants to stay in the lowest state of entropy. Bargain et al. describe two types of defects, one are the permanent defects, which are these $\mathrm{H}-\mathrm{H}$ and $\mathrm{T}$ - $\mathrm{T}$ based defects that cannot be removed. The other are the imperfect crystals, which might be formed due to quenching (temporary defects) and could be removed after repeated annealing [60]. 
To summarize the argument of T.R.V.: Though the permanent defects cannot be removed from the polymer system, they could be still removed from the primary crystal structure. Therefore, in this case, the chemical defects might not play a role in the mid-temperature transition, as it is clearly a structural effect, which might be connected to the temporary defects described in the beginning. They can be removed by means of a thermal treatment, as shown in the above DSC results for the second heating cycle (Figure 7 and 8).

There was a question raised whether there are any domainwall motions or domain-wall relaxations going in polymers [62]. According to B.P., domains in semi-crystalline polymers are more difficult to explain than those in inorganic crystalline materials. It is to be noted that in semi-crystalline polymers, a variety of crystal structures can be present. In PVDF, we normally have spherulitic structures the size of which is in the micrometer range and depends on various factors such as crystallization temperature, time and other kinetics [63]. The spherulites in turn contain regularly arranged molecular folds called lamella. Theoretically, it has been estimated that a fold contains about 30 repeat units and that the typical lamellar thicknesses range from about 50 to approximately 130 repeat units [48, 65]. On stretching, the lamellar thicknesses in the spherulites can be drastically reduced. Also, in the past, single crystals of PVDF have been prepared [65]. The crystallite sizes and size distributions vary with the respective co- or termonomer [67, 68]. Especially for relaxor-ferroelectric terpolymers such as P(VDF-TrFE-CFE), the crystalline regions are reduced to a nanometer scale $[69,70]$. In addition to all these factors, the presence of amorphous phase and of an interface with the crystalline phase complicates the situation. This makes it very difficult to identify domains in VDF-based materials. Hence, B.P. explained that in polymers we have crystallites that are not too large - say $20 \mathrm{~nm}$ in diameter. When the material is not polarized, we know that one crystallite falls into a domain because it is smaller, but B.P. acknowledged that he does not know if domain-wall motion has been experimentally observed in polymers. In addition, M.W. mentioned topological defects in semi-crystalline polymers, which are expelled from the crystals, and there is no way for them to get back into the crystal, and in this way, you obscure some motion and switching locally. If the electric fields are high enough and if we have not so many of the topological defects, we can have mono-domains at high fields and this is probably not the most relevant thing here. So, according to M.W., domain-wall motion does not have a role in the mid-temperature transition. T.F. added that domain walls can move thermally and that we can observe the motion, and that we have switching in terms of domain-wall motion. We also have field-induced domain motion. Thus, most of the domain-wall motion can be observed.

M.W. asked T.F. about the torsional or rotational motion of chains that he had mentioned previously. Under the assumption of oriented all-trans chains, we know now from T.F. that the motional process goes all the way into the melt without substantial changes in the activation energy. This observation is most intriguing since it implies that the dynamics in the (plastic) crystalline phase - the paraelectric phase - involves nearly the same motional degrees of freedom and energetic barriers as in the melt, where this relaxation must represent the primary (structural) relaxation. Following this idea, M.W. wondered how the relaxation spectrum in the melt exactly looks like. Apart from expected discontinuities in the spectral shape parameters (e.g. the slopes $m$ and $n$ in the Havriliak-Negami function) and in the relaxation strength (due to the unlocking of all rotational degrees of freedom and possible changes in the dipole-dipole correlation coefficient), one should check whether the shape parameters obey the typical scaling laws [70] predicting a "Debye-like" slope $(m \approx 1)$ at the low-frequency side and a high-frequency slope $n \rightarrow 0.5$ that results from the chain-connectivity (conformation diffusion) in the high temperature limit (also described by the Cole-Davidson function). Deviations from the scaling predictions based on BDS measurements in the RF- range $\left(10^{7} \leq f \leq 10^{10} \mathrm{~Hz}\right)$ would be a strong hint for the existence of structures in the melt such as rigid polar clusters (RPCs, as e.g. observed in glycerol melts, [71]) causing a true Debye-like response, mesomorphic structures as found for PEN above $T_{m}$ [72] or other peculiarities.

T.F. replied that we also have segmental motion in the amorphous regions. Amorphous regions do not mean a supercooled state. When you cool from the melt state, the amorphous regions do not crystallize and stay there. As a result, an amorphous region is a liquid-like region in the melt, and molecular motion should be also continuing even after it has been cooled. It is a different story in the case of crystalline motion. T.F. had used almost $100 \%$ crystalline samples (highly stretched samples) and observed dipole relaxations and elastic relaxations. So, how to interpret this motion in the melt phase? The answer is that we need to draw a good picture. One must have a very good imagination to describe it. Crystalline motions arise from some local motions if they expand in the chain direction. What we clearly show experimentally is that the crystalline region is causing molecular motion.

It was stated that inorganic ferroelectric materials show very similar relaxation spectra as you see on semi-crystalline polymers, and they are always explained by domains [73]. This does not mean that these are always domains. We explain everything with domains because we do not know anything better. It was suggested to do temperature-dependent relaxation spectra of the VDF-based polymers with an electric-field bias applied, as we normally do on inorganic ferroelectrics. Sometimes the relaxations are suppressed, or you can see whether the transitions are from structural processes or from defects. B.P. concluded with the statement that the problem has not been solved, but that the discussion has provided lots of useful hints where to continue the research.

The summarize, the panel discussion at the 17 th International Symposium on Electrets (ISE-17) in Limerick, Ireland started with an introductory presentation by Thulasinath Raman Venkatesan (T.R.V.) who spoke about the mystery surrounding the origin of the mid-temperature transition in vinylidene fluoride-based homo-, co- and ter-polymers. The various explanations commonly found in the literature were stated followed by the hypothesis of T.R.V. in which he suggested that the mid-temperature transition is a result of several interrelated processes taking place over a certain temperature range. This was followed by the presentation of Michael Wübbenhorst who suggested that the frequency-independent peaks which he had 
obtained from his derivative analysis of the dielectric data of unstretched and stretched P(VDF-TFE) films points to a structural transition affecting the fluctuation angle of molecular motions. He also hinted at the possibility of conformational disorder playing an important role for this transition. Later during the discussion, he mentioned the possible role of secondary crystals or nematic structures with high order at least in the case of thin films.

Takashi Nakajima used DSC scans of P(VDF-TrFE) powders to show that the mid-temperature transition is not present in the powder state of the copolymer, indicating the possible role of processing and of residual solvent. Then, Xunlin Qiu spoke in detail about the effect of poling on P(VDF-TrFE) copolymers and stated the possibility of studying its effects on the midtemperature transition especially from the point of view of Maxwell-Wagner interface polarization which could be influenced by the poling field. Bernd Ploss also spoke from the polarization point of view using his previous results from nonlinear dielectric measurements where he has also observed a similar mid-temperature transition in $\mathrm{P}(\mathrm{VDF}-\mathrm{TrFE})$ thin films.

Takeo Furukawa, this year's Bernhard Gross Memorial Lecturer, also participated in the lively discussion and mentioned the various relaxation processes in $\alpha$ - and $\beta$-phase PVDF. He said that the $\alpha$ relaxation (relaxation associated with $\left.T_{\text {mid }}\right)$ is related to the transitional motion in the semi-crystalline regions. He also mentioned that at room temperature, the $\beta$ and $\gamma$ relaxations overlap and cannot be distinguished, extending very well into the melt of the sample. Hence, he suggested that by looking into the complete spectrum from very low frequencies $(<10 \mathrm{mHz})$ to very high frequencies $(\mathrm{GHz})$ and from very low temperatures to high temperatures, one can identify all the molecular processes taking place within the material, which will help to better understand the origin behind the mid-temperature transition.

\section{CONCLUSIONS}

In this review, a brief history of pyro-, piezo- and ferroelectricity in dielectric materials and in particular in VDFbased polymers was provided together with a summary of the essential structural and morphological features of PVDF and some of its co- and ter-polymers. This led into a presentation of the often observed mid-temperature transition in VDF-based polymers and its several different interpretations and explanations. A new hypothesis was put forward in which the mid-temperature transition is considered as a result of several independent, but subsequent processes occurring in the same temperature range. According to the hypothesis, when the sample is heated above the conventional glass-transition temperature, the constrained amorphous regions embedded between crystallites relax leading to the observation of an upper glass transition. Followed by this, the $T G^{+} T G^{-}$chains both in the $\alpha$-crystals and in the amorphous regions undergo conformational-disorder-related (condis) relaxation where the fluorine atoms flips from up to down or vice versa $\left(T G^{+} T G^{-} \rightarrow\right.$ $\left.G^{-} T G^{+} T\right)$. Finally, on further heating, the secondary crystals which are formed as a result of the storage or cooling history melt leading to an often observed endothermic peak. In addition, charges injected during poling are also expected to play a definitive role in the transition-as evident from the peaks in the TSDC curves. As all these mechanisms occur in a very small temperature range, some of them are masked/overlapped when observed using different experimental techniques.

On the basis of the hypothesis, it has been shown to which extent the effect of each individual mechanism is enhanced/reduced for a given set of processing conditions of the material under study. From DSC measurements, it is seen that stretching the sample at low temperatures, after quenching from the melt, yields an endothermic peak, which indicates a major contribution from the melting of secondary crystals. This is further elucidated from the derivative analysis of DRS measurements where a frequency-independent peak is clearly seen in the mid-temperature range pointing out to a structural transition such as the melting of secondary crystals or nematic structures. In samples annealed or/and stretched at high temperatures and also in samples slowly cooled from their respective melt state, the mid-temperature transition manifests as a relaxation process dominated by condis. In all cases, as seen from the DSC curves, the transition disappears in the second heating cycle, but is always present in the cooling cycles. Also, $T_{\text {mid }}$ peaks observed in unstretched samples from DRS measurements either disappear or are suppressed as a result of stretching. Hence, processing clearly affects the dynamics of the mid-temperature transition. On the other hand, PVDF homopolymer and its copolymer with TrFE which are highly crystalline shows the lowest sensitivity to this midtemperature transition. When it comes to P(VDF-TFE) copolymer due to the presence of lower crystallinity, and $\mathrm{P}(\mathrm{VDF}-\mathrm{TrFE}-\mathrm{CFE})$ terpolymer both due to its lower crystallinity and higher amorphous-crystalline (a-c) interface area shows a strong sensitivity towards mid-temperature transition. It should be noted that the transition is also affected by the crystalline, amorphous phases and its interface. This can also be inferred from the absence of a mid-temperature peak/relaxation step in the DSC curve of a P(VDF-TrFE) copolymer in its powder state where the interaction of the crystalline phase within itself and also with the a-c interface is expected to be less.

The role of electrical poling and polarization on the midtemperature peak is seen from the hysteresis and non-linear dielectric spectroscopy measurements on P(VDF-TrFE) copolymer. It has been shown that the remanent polarization $\left(P_{r}\right)$ increases with increase in the number of poling cycles suggesting a change in crystallinity and also the polar phase content in the copolymer which in turn can affect the midtemperature transition. The presence of a peak like structure in the mid-temperature range around $60{ }^{\circ} \mathrm{C}$ in the plot of $\varepsilon_{2} /\left(3 \varepsilon_{0}^{2} \varepsilon_{1}^{3}\right)$ (a ratio proportional to $\left.P_{r}\right)$ versus temperature clearly shows the role of the electrical polarization in this transition. In addition, the presence of a persistent $P_{r}$ which depends on the first poling direction and only removed by dissolving the film indicates the possible role of a-c interface and its related structures, and to presence of Maxwell-Wagner polarization.

All the above-mentioned factors probably contribute to the fact that different authors in the past observed quite different results in their experiments-depending on the particular VDFbased material system and on the processing conditions they 
had used to study the transition. However, when putting the pieces of the puzzle together by considering all these factors, the heterogeneity of the transition becomes obvious, and hence the hypothesis of multiple origins becomes the most plausible explanation.

From the proposed hypothesis and the new insights gained from the experiments performed in this work, we now have a better understanding of the mid-temperature transition. This can help to further understand the impact of the mid-temperature transition on applications-relevant properties such as aging, shrinkage, decay of piezoelectricity and Curie-transition temperature. By studying in detail how the mid-temperature transition is affected by environmental factors (temperature, pressure, etc.), we can achieve better control/tailoring of the mid-temperature transition with regard to the specific application in which a VDF-based polymer is used. In this context, measuring the dielectric response over a broad frequency range and at higher temperatures in the melt would be helpful - as suggested during the discussion. Also, additional non-linear dielectric measurements and the use of electric bias fields during temperature-dependent dielectric measurements could shed additional light on the intriguing phenomenon that has been observed many times, but apparently never been explained conclusively before.

\section{ACKNOWLEDGMENTS}

Thulasinath Raman Venkatesan would like to thank all the panelists for kindly accepting the invitation to contribute to the discussion and to Bernd Ploss for also serving as moderator of the discussion. The authors are very grateful to the organizers and the scientific committee of ISE-17 for arranging and supporting the panel discussion. In particular, we are indebted to the chairman of ISE-17, Prof. Tofail Syed, for the invitation to contribute the present review and for his constant support and encouragement. We wish to thank Colette Lacabanne (University of Toulouse), Tahsin Morshed (BUET Dhaka) and Dragan Damjanovic (EPFL Lausanne), all members of the audience, for their stimulating comments and suggestions during the panel discussion. Special thanks are due to Sarah Markham (University of Limerick for the arrangements to record the discussion and to Anna A. Gulyakova (HSPU St. Petersburg) for providing the P(VDF-TFE) samples used in the experimental study of the mid-temperature transition.

\section{REFERENCES}

[1] H. Kawai, "The Piezoelectricity of Poly (vinylidene Fluoride)," Japanese Journal of Applied Physics, vol. 8, no. 7, p. 975, 1969, doi: 10.1143/JJAP.8.975.

[2] S. B. Lang, "Pyroelectricity: From Ancient Curiosity to Modern Imaging Tool," Physics Today, vol. 58, no. 8, pp. 31-36, 2005, doi: 10.1063/1.2062916.

[3] J. Curie and P. Curie, “Développement par compression de l'électricité polaire dans les cristaux hémièdres à faces inclinées," (Development of polar electricity through compression in hemihedral crystals at inclined faces), Bulletin de la Société minéralogique de France, vol. 3, no. 4, pp. 90-93, 1880, doi: 10.3406/bulmi.1880.1564.

[4] S. Katzir, The Beginnings of Piezoelectricity: A Study in Mundane Physics. Springer Netherlands, 2006.

[5] J. Valasek, "The early history of ferroelectricity," Ferroelectrics, vol. 2, no. 1, pp. 239-244, 1971, doi: 10.1080/00150197108234098.
[6] H. Staudinger, "Über Polymerisation," Berichte der deutschen chemischen Gesellschaft (A and B Series), vol. 53, no. 6, pp. 10731085, 1920, doi: 10.1002/cber.19200530627.

[7] E. Fukada, "History and recent progress in piezoelectric polymers," IEEE Transactions on Ultrasonics, Ferroelectrics and Frequency Control, vol. 47, no. 6, pp. 1277-1290, 2000, doi: 10.1109/58.883516.

[8] J. G. Bergman Jr., J. H. McFee, and G. R. Crane, "Pyroelectricity and Optical Second Harmonic Generation in Polyvinylidene Fluoride Films", Applied Physics Letters, vol. 18, no. 5, pp. 203-205, 1971; doi: 10.1063/1.1653624.

[9] T. Furukawa, M. Date, and E. Fukada, "Hysteresis phenomena in polyvinylidene fluoride under high electric field," Journal of Applied Physics, vol. 51, no. 2, pp. 1135-1141, 1980, doi: 10.1063/1.327723.

[10] M. Eguchi, "On Dielectric Polarisation," Proc. of the PhysicoMathematical Society of Japan. 3rd Series, vol. 1, 1919, no. 10-11, pp. 326-331, doi: 10.11429/ppmsj1919.1.10-11_326.

[11] M. Eguchi, "XX. On the permanent electret," The London, Edinburgh, and Dublin Philosophical Magazine and Journal of Science, vol. 49, no. 289, pp. 178-192, 1925, doi: 10.1080/14786442508634594.

[12] B. Gross, "The Electret," Endeavour (ICI), vol. 30, no. 111, pp. 115119, 1971, doi: 10.1016/0160-9327(71)90036-6.

[13] N. Murayama, "Persistent polarization in poly(vinylidene fluoride). I. Surface charges and piezoelectricity of poly(vinylidene fluoride) thermoelectrets," Journal of Polymer Science: Polymer Physics Edition, vol. 13, no. 5, pp. 929-946, 1975, doi: 10.1002/pol.1975.180130505.

[14] N. Murayama, T. Oikawa, T. Katto, and K. Nakamura, "Persistent polarization in poly(vinylidene fluoride). II. Piezoelectricity of poly(vinylidene fluoride) thermoelectrets," Journal of Polymer Science: Polymer Physics Edition, vol. 13, no. 5, pp. 1033-1047, 1975, doi: 10.1002/pol.1975.180130515.

[15] N. Murayama and H. Hashizume, "Persistent polarization in poly(vinylidene fluoride). III. Depolarization and pyroelectricity of poly(vinylidene fluoride) thermoelectrets," Journal of Polymer Science: Polymer Physics Edition, vol. 14, no. 6, pp. 989-1003, 1976, doi: 10.1002/pol.1976.180140603.

[16] T. Furukawa, "Recent advances in ferroelectric polymers," Ferroelectrics, vol. 104, no. 1, pp. 229-240, 1990, doi: 10.1080/00150199008223826.

[17] G. M. Sessler et al., "Piezo- and Pyroelectricity in Electrets - Charges or Dipoles or Both?," IEEE Transactions on Electrical Insulation, vol. 27, no. 1, pp. 872-897, 1992, doi: 10.18419/opus-4986.

[18] G. Eberle, H. Schmidt, and W. Eisenmenger, "Piezoelectric polymer electrets," IEEE Transactions on Dielectrics and Electrical Insulation, vol. 3, no. 5, pp. 624-646, 1996, doi: 10.1109/94.544185.

[19] A. J. Lovinger, "Ferroelectric Polymers," Science, vol. 220, no. 4602, pp. 1115-1121, 1983, doi: 10.1126/science.220.4602.1115.

[20] D. K. Das-Gupta and K. Doughty, "Corona charging and the piezoelectric effect in polyvinylidene fluoride," Journal of Applied Physics, vol. 49, no. 8, pp. 4601-4603, 1978, doi: 10.1063/1.325441.

[21] Lovinger, Andrew J., "Annealing of poly (vinylidene fluoride) and formation of a fifth phase," ACS Publications, vol. 15, no. 1, pp. 4044, 1982, doi: 10.1021/ma00229a008.

[22] T. Furukawa, "Ferroelectric properties of vinylidene flu-oride copolymers," Phase Transitions, vol. 18, no. 3-4, pp. 143-211, 1989, doi: 10.1080/01411598908206863.

[23] A. C. Jayasuriya, A. Schirokauer, and J. I. Scheinbeim, "Crystalstructure dependence of electroactive properties in differently prepared poly(vinylidene fluoride/hexafluoropropylene) copolymer films," Journal of Polymer Science Part B: Polymer Physics, vol. 39, no. 22, pp. 2793-2799, 2001, doi: 10.1002/polb.10035.

[24] Odajima A, Takase Y, Ishibashi T, and Yuasa K, "Irradiation effects on the ferroelectric phase transition of vinylidene fluoride and trifluoroethylene copolymers," Proc. of 5th International Symposium on Electrets (ISE), 1985,pp.642-647,doi: 10.1109/ISE.1985.7341550.

[25] Q. M. Zhang, "Giant Electrostriction and Relaxor Ferroelectric Behavior in Electron-Irradiated Poly(vinylidene fluoridetrifluoroethylene) Copolymer," Science, vol. 280, no. 5372, pp. 21012104, 1998, doi: 10.1126/science.280.5372.2101.

[26] H. Xu, Z.-Y. Cheng, D. Olson, T. Mai, Q. M. Zhang, and G. Kavarnos, "Ferroelectric and electromechanical pro-perties of poly(vinylidene fluoride-trifluoroethylene-chlorotrifluoroethylene) terpolymer," Applied Physics Letters, vol. 78, no. 16, pp. 2360-2362, 2001, doi: 10.1063/1.1358847. 
[27] F. Xia, "High Electromechanical Responses in a Poly(vinylidenefluoride-trifluoro-ethylene-chlorofluoro-ethylene) Terpolymer," Advanced Materials, vol. 14, no. 21, pp. 1570-1574, 2002.

[28] L. Zhu, "Exploring Strategies for High Dielectric Constant and Low Loss Polymer Dielectrics," The Journal of Physical Chemistry Letters, vol. 5, no. 21, pp. 3677-3687, 2014, doi: 10.1021/jz501831q

[29] H. Sasabe, S. Saito, M. Asahina, and H. Kakutani, "Dielectric relaxations in poly(vinylidene fluoride)," Journal of Polymer Science Part A-2: Polymer Physics, vol. 7, no. 8, pp. 1405-1414, 1969, doi: 10.1002/pol.1969.160070810.

[30] S. Yano, "Dielectric relaxation and molecular motion in poly(vinylidene fluoride)," Journal of Polymer Science Part A-2: Polymer Physics, vol. 8, no. 7, pp. 1057-1072, 1970, doi: 10.1002/pol.1970.160080704.

[31] P. Frübing, F. Wang, and M. Wegener, "Relaxation pro-cesses and structural transitions in stretched films of poly vinylidenefluoride and its copolymer with hexafluoro-propylene," Applied Physics A, vol. 107, no. 3, pp. 603-611, Jun. 2012, doi: 10.1007/s00339-012-6838-1.

[32] R. F. Boyer, "Glassy transitions in semicrystalline polymers," Journal of Polymer Science: Polymer Symposia, vol. 50, no. 1, pp. 189-242, 1975, doi: 10.1002/polc.5070500114.

[33] C. Leonard, J. L. Halary, L. Monnerie, and F. Micheron, "DSC studies on the transitions in poly(vinylidenefluoride) and some related copolymers," Polymer Bulletin, vol. 11, no. 2, 1984, doi: 10.1007/BF00258030.

[34] J. B. Enns and R. Simha, "Transitions in semicrystalline polymers. I. poly(vinyl Fluoride) and poly(vinylidene fluoride)," Journal of Macromolecular Science, Part B, vol. 13, no. 1, pp. 11-24, 1977, doi: 10.1080/00222347708208750..

[35] G. Teyssedre, M. Grimau, A. Bernes, J. J. Martinez, and C. Lacabanne, " $\alpha$-relaxation/retardation mode in semicrystalline polymers with flexible chains," Polymer, vol. 35, no. 20, pp. 4397-4403, 1994, doi: 10.1016/0032-3861(94)90098-1.

[36] M. Neidhöfer, F. Beaume, L. Ibos, A. Bernès, and C. Lacabanne, "Structural evolution of PVDF during storage or annealing," Polymer, vol. 45, no. 5, pp. 1679-1688, 2004, doi: 10.1016/j.polymer.2003.12.066.

[37] Miyamoto, H. Miyaji, and K. Asai, "Anisotropy of dielectric relaxation in crystal form II of poly(vinylidene fluoride)," Journal of Polymer Science: Polymer Physics Edition, vol. 18, no. 3, pp. 597-606, 1980, doi: 10.1002/pol.1980.180180318.

[38] K. Loufakis and B. Wunderlich, "Thermal analysis of the conformational disorder in semicrystalline poly(vinylidene fluoride) and poly(trifluoroethylene)," Macromolecules, vol. 20, no. 10, pp. 2474-2478, 1987, doi: 10.1021/ma00176a026.

[39] D. Rollik, S. Bauer, and R. Gerhard(-Multhaupt), "Separate contributions to the pyroelectricity in poly(vinylidene fluoride) from the amorphous and crystalline phases, as well as from their interface," Journal of Applied Physics, vol. 85, no. 6, pp. 3282-3288, 1999, doi: 10.1063/1.369672.

[40] T. Raman Venkatesan, A. A. Gulyakova, P. Frübing, and R. Gerhard, "Electrical polarization phenomena, dielectric relaxations and structural transitions in a relaxor-ferroelectric terpolymer investigated with electrical probing techniques," Materials Research Express, vol. 6 , no. 12, p. 125301, 2019, doi: 10.1088/2053-1591/ab5352.

[41] L. L. Blyler, G. E. Johnson, and N. M. Hylton, "Characterization of biaxially-oriented polyvinylidene fluoride-film for transducer applications," Ferroelectrics, vol. 28, no. 1, pp. 303-306, 1980, doi: $10.1080 / 00150198008227094$.

[42] A. G. Kolbeck, "Aging of piezoelectricity in poly(vinylidene fluoride)," Journal of Polymer Science: Polymer Physics Edition, vol. 20, no. 11, pp. 1987-2001, 1982, doi: 10.1002/pol.1982.180201102.

[43] G. Teyssèdre and C. Lacabanne, "Study of the thermal and dielectric behavior of $\mathrm{P}(\mathrm{VDF}-\mathrm{TrFE})$ copolymers in relation with their electroactive properties," Ferroelectrics, vol. 171, no. 1, pp. 125-144, 1995, doi: 10.1080/00150199508018427.

[44] T. Raman Venkatesan and R. Gerhard, "Origin of the mid-temperature transition in vinylidenefluoride-based ferro-, pyro- and piezoelectric homo-, co- and ter-polymers," Materials Research Express, 2020, doi: $10.1088 / 2053-1591 / \mathrm{ab} 842 \mathrm{c}$.

[45] M. Wübbenhorst and J. van Turnhout, "Analysis of complex dielectric spectra. I. One-dimensional derivative techniques and threedimensional modelling," Journal of Non-Crystalline Solids, vol. 305, no. 1, pp. 40-49, 2002, doi: 10.1016/S0022-3093(02)01086-4.
[46] J. van Turnhout and M. Wübbenhorst, "Analysis of complex dielectric spectra. II: Evaluation of the activation energy landscape by differential sampling," Journal of Non-Crystalline Solids, vol. 305, no. 1, pp. 5058, 2002, doi: 10.1016/S0022-3093(02)01120-1

[47] K. Nakagawa and Y. Ishida, "Annealing effects in poly(vinylidene fluoride) as revealed by specific volume measurements, differential scanning calorimetry, and electron microscopy," Journal of Polymer Science: Polymer Physics Edition, vol. 11, no. 11, pp. 2153-2171, 1973, doi: 10.1002/pol.1973.180111107.

[48] S. Bauer and F. Bauer, "Piezoelectric Polymers and Their Applications," in Piezoelectricity: Evolution and Future of a Technology, W. Heywang, K. Lubitz and W. Wer-sing, Springer, Berlin, Heidelberg, 2008, pp. 157-177.

[49] F. Bauer and R. A. Graham, "Very high pressure behavior of preciselypoled PVDF," Ferroelectrics, vol. 171, no. 1, pp. 95-102, 1995, doi: 10.1080/00150199508018424.

[50] Q. D. Nguyen, T. R. Venkatesan, W. Wirges, and R. Gerhard, "Nonuniform polarization profiles in $\mathrm{P}(\mathrm{VDF}-\mathrm{TrFE})$ copolymer films after cyclical poling," in Proc. IEEE International Symposium on Applications of Ferroelectrics (ISAF), 2019, pp. 1-4, doi: 10.1109/ISAF43169.2019.9034937.

[51] D. Nordheim, S. Hahne, and B. Ploss, "Nonlinear dielectric properties and polarization in thin ferroelectric $\mathrm{P}(\mathrm{VDF}-\mathrm{TrFE})$ copolymer films," IEEE Transactions on Dielectrics and Electrical Insulation, vol. 19, no. 4, pp. 1175-1180, 2012, doi: 10.1109/TDEI.2012.6259986.

[52] B. Ploss, "Influence of poling and annealing on the nonlinear dielectric permittivity of PVDF-TRFE copolymers," IEEE Transactions on Dielectrics and Electrical Insulation, vol. 5, no. 1, pp. 91-95, 1998, doi: 10.1109/94.660777.

[53] A.J. Lovinger "Poly(vinylidene Fluoride)" in Developments in Crystalline Polymers-1, vol. 33, D.C. Bassett,Ed. Springer, 1982, 195-261.

[54] A. Salimi and A. A. Yousefi, "Conformational changes and phase transformation mechanisms in PVDF solution-cast films," Journal of Polymer Science Part B: Polymer Physics, vol. 42, no. 18, pp. 34873495, 2004, doi: 10.1002/polb.20223.

[55] Y. Nabata, "Molecular Motion in Form II Poly(vinylidene Fluoride)," Japanese Journal of Applied Physics, vol. 29, no. Part 1, No. 12, pp. 2782-2788, 1990, doi: 10.1143/JJAP.29.2782.

[56] T. Furukawa, Y. Tajitsu, X. Zhang, and G. E. Johnson, "Dielectric relaxations in copolymers of vinylidene fluoride," Ferroelectrics, vol. 135, no. 1, pp. 401-417, 1992, doi: 10.1080/00150199208230041.

[57] T. Raman Venkatesan, A. A. Gulyakova, P. Frubing, and R. Gerhard, "Relaxation processes and structural transitions in Poly(vinylidene fluoride-trifluoroethylene-chlorofluoroethylene) relaxor-ferroelectric terpolymers as seen in dielectric spectroscopy," IEEE Transactions on Dielectrics and Electrical Insulation, vol. 25, no. 6, pp. 2229-2235, 2018, doi: 10.1109/TDEI.2018.007440.

[58] G. Teyssèdre and C. Lacabanne, "Study of the thermal and dielectric behavior of $\mathrm{P}(\mathrm{VDF}-\mathrm{TrFE})$ copolymers in relation with their electroactive properties," Ferroelectrics, vol. 171, no. 1, pp. 125-144, 1995, doi: 10.1080/00150199508018427.

[59] C. Lacabanne, Public communication during the panel discussion at the $17^{\text {th }}$ International Symposium on Electrets (ISE-17), Limerick, Ireland, September 2019.

[60] F F. Bargain, P. Panine, F. Domingues Dos Santos, and S. TencéGirault, "From solvent-cast to annealed and poled poly(VDF- co -TrFE) films: New insights on the defective ferroelectric phase," Polymer, vol. 105, pp. 144-156, 2016, doi: 10.1016/j.polymer.2016.10.010.

[61] K. Tashiro and M. Kobayashi, "Structural phase transition in ferroelectric fluorine polymers: X-ray diffraction and infrared/Raman spectroscopic study," Phase Transitions, vol. 18, no. 3-4, pp. 213-246, 1989, doi: 10.1080/01411598908206864.

[62] T. Morshed, Public communication during the panel discussion at the $17^{\text {th }}$ International Symposium on Electrets (ISE-17), Limerick, Ireland, September 2019.

[63] M. P. Silva et al., " $\alpha$ - and $\gamma$-PVDF: Crystallization ki-netics, microstructural variations and thermal behavior," Materials Chemistry and Physics, vol. 122, no. 1, pp. 87-92, 2010, doi: 10.1016/j.matchemphys.2010.02.067.

[64] K. Nakagawa and Y. Ishida, "Dielectric relaxations and molecular motions in poly(vinylidene fluoride) with crystal form II," Journal of Polymer Science Part A-2: Polymer Physics, vol. 11, no. 8, pp. 15031533, 1973, doi: 10.1002/pol.1973.180110804. 
[65] A. Toda, T. Arita, and M. Hikosaka, "Three-dimensional morphology of PVDF single crystals forming banded spherulites," Polymer, vol. 42, no. 5, pp. 2223-2233, 2001, doi: 10.1016/S0032-3861(00)00446-8.

[66] H.-M. Bao, J.-F. Song, J. Zhang, Q.-D. Shen, C.-Z. Yang, and Q. M. Zhang, "Phase Transitions and Ferroelectric Relaxor Behavior in P(VDF-TrFE-CFE) Terpolymers," Macromolecules, vol. 40, no. 7, pp. 2371-2379, 2007, doi: 10.1021/ma0628001.

[67] T. Raman Venkatesan, P. Frübing, and R. Gerhard, "Influence of Composition and Preparation on Crystalline Phases and Morphology in Poly(vinylidene fluoride-trifluoroethylene-chlorofluoroethylene) Relaxor-Ferroelectric Terpolymer," in Proc. IEEE $2^{\text {nd }}$ International Conference on Dielectrics (ICD), 2018, pp. 1-4, doi: 10.1109/ICD.2018.8468492.

[68] F. Bauer, E. Fousson, Q. M. Zhang, and L. M. Lee, "Ferroelectric copolymers and terpolymers for electrostrictors: synthesis and properties," IEEE Transactions on Dielectrics and Electrical Insulation, vol. 11, no. 2, pp. 293-298, 2004, doi: 10.1109/TDEI.2004.1285900.

[69] T. C. Chung and A. Petchsuk, "Synthesis and Properties of Ferroelectric Fluoroterpolymers with Curie Transition at Ambient Temperature," Macromolecules, vol. 35, no. 20, pp. 7678-7684, 2002, doi: $10.1021 / \mathrm{ma} 020504 \mathrm{c}$.

[70] A. Schönhals, F. Kremer, and E. Schlosser, "Scaling of the $\alpha$ relaxation in low-molecular-weight glass-forming liquids and polymers," Physical Review Letters, vol. 67, no. 8, pp. 999-1002, 1991, doi: 10.1103/PhysRevLett.67.999.

[71] A. Kasina, T. Putzeys, and M. Wübbenhorst, "Dielectric and specific heat relaxations in vapor deposited glycerol," The Journal of Chemical Physics, vol. 143, no. 24, p. 244504, 2015, doi: 10.1063/1.4937795.

[72] M. Wübbenhorst, "'Direct evidence of coexisting amorphous, mesomorphic and crystalline phases in PEN from dielectric spectroscopy," Abstracts of Papers of the American Chemical Society, PMSE, vol. 97, pp. 829-830, 2007. Crystalline Phases in PEN from Dielectric Spectroscopy," p. 2

[73] D. Damjanovic, Public communication during the panel discussion at the $17^{\text {th }}$ International Symposium on Electrets (ISE-17), Limerick, Ireland, September 2019.

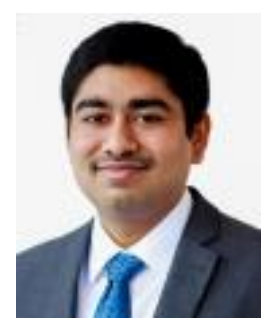

Thulasinath Raman Venkatesan was born in Chennai, India in 1992. He studied Plastics Technology at the Central Institute of Plastics Engineering and Technology (CIPET), Govt. of India, in Chennai and received his Bachelor-in-Technology (B.Tech) degree with a University rank in 2014. He worked as a Graduate Engineer in the plastics industry in Chennai from 2014 to 2015 in the area of plastics processing, marketing and technology services. He holds a master's degree from the Joint Master-ofScience Program in Polymer Science of the four universities in Berlin and Potsdam. He is currently doing his doctoral studies at the University of Potsdam and KU Leuven. His research interests include the investigation of dielectric, electromechanical and energy-storage properties of VDF-based homo-, co- and ter-polymers and of other ferroelectric polymers.

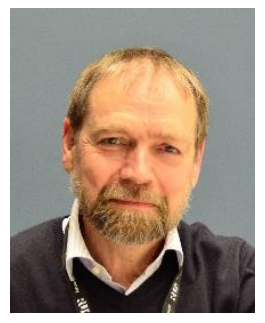

Michael Wübbenhorst was born in Stendal, Germany in 1958. He received his Master and Ph.D. degrees in physics from the University of Leipzig in 1985 and 1989 respectively. In 1990, he joined the Delft University of Technology in The Netherlands as a post-doctoral researcher. In 1995, he became assistant professor in the polymer group at TU Delft. From his position as associate professor (2004) he moved to Belgium where he was appointed at the KU Leuven as professor in experimental soft matter physics in 2005. Since 2014 he is head of the Laboratory for Soft Matter and Biophysics (formerly ATF). His scientific interest covers soft matter physics with emphasis on glass forming materials, functional polymers and liquid as well as molecular crystals. He is a member of the German Physical Society (DPG), American Chemical Society (ACS), Belgian Physical Society, IEEE, and a board member of the International Dielectric Society (IDS) and serves in the scientific advisory committees of various international conferences.

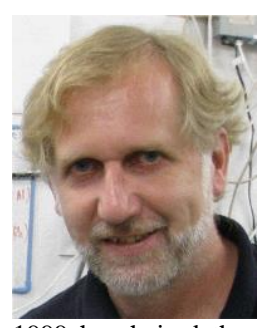

Bernd Ploss was born in Calw, Germany in 1955. From the University Karlsruhe he was awarded the Diploma in physics with distinction in 1981 and the Ph.D. (Dr. rer. nat.) degree with distinction in 1987. In 1997 he habilitated and received the title Privatdozent. In 1997 he joined the Hong Kong Polytechnic University where he became an Associate Professor in 1999. Since 2001 he is with the University of Applied Sciences Jena, Germany as a Professor for Physics Instrumentation and Solid State Physics. From 1995 to 1999 he chaired the section "Dielectric Materials" of the German Physical Society (DPG). His main interests are materials physics, dielectric phenomena and ferroelectrics.

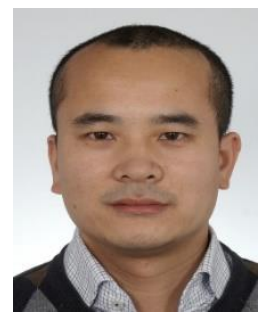

Xunlin Qiu was born in 1978. He received the Ph.D. degree in condensed matter physics from the Pohl Institute of Solid State Physics, Tongji University, in 2006. He then joined the group of Applied-Condensed Matter Physics at the University of Potsdam as a postdoc. From 2007 to 2009, he held a DFG (German Research Foundation) research fellowship. He then became a Senior Staff Member with the University of Potsdam, where he received the Habilitation (highest German university degree) in 2017. Since 2017, he has been a Senior Staff Member with the Institute for Print and Media Technology, Chemnitz University of Technology. He has authored or co-authored two book chapters and over 100 scientific journal and conference papers. His main research work is dedicated to functional dielectrics and electrets, to piezoelectricity in polar polymer films and non-polar polymer foams and to applications of dielectrics and electrets.

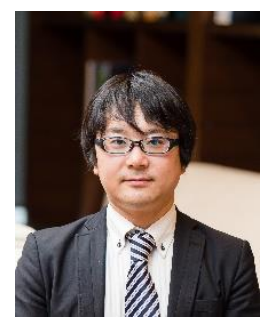

Takashi Nakajima received his B.Sci., M.Sci., and Ph.D. degrees from Tokyo University of Science, Tokyo, Japan, in 2003, 2005, and 2008, respectively. In 2008-2012, he worked at Tokyo University of Science as a research associate. In 2012-2014, he was in Institute of Materials Research, Tohoku University as a research associate. Currently, he is an associate professor in the Department of Applied Physics, Faculty of Science, Tokyo University of Science. He holds the concurrent post of PRESTO researcher of Japan Science and Technology Agency. His research has been focused on the functional properties of ferroelectric materials and the ferroelectric devices including piezoelectric energy harvester.

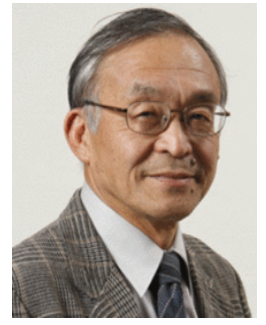

Takeo Furukawa received the B.S., M.S. and Ph.D. degrees in applied physics from the University of Tokyo. In 1968-1992 he worked for the Institute of Physical and Chemical Research. Since 1992, he has been a member of the Department of Chemistry, the Faculty of Science, Tokyo University of Science where he is currently a professor. His research has been focused on the functional properties of soft materials including ferroelectric, piezoelectric, pyroelectric and conducting polymers and composites.

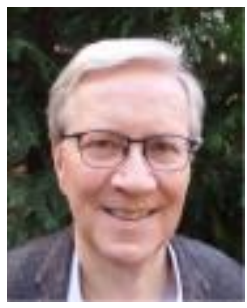

Reimund Gerhard was born in Heidelberg, Germany in 1952. He studied mathematics and physics at the Technical University of Darmstadt. After a research fellowship at the Collège Militaire Royal in Saint-Jean, Québec, Canada, he obtained his Ph.D. with Gerhard Sessler in Darmstadt in 1984. From 1985 until 1994, he was project manager at the Heinrich-Hertz Institute (HHI) in Berlin. In 1994, he was appointed professor at the University of Potsdam. His team works on the preparation, investigation and application of dielectric elastomers (electro-electrets), polymeric space-charge electrets, polymer ferroor piezoelectrets, ferro-, pyro- and piezoelectric polymers and polymer composites, and on acoustical studies of musical instruments with minimally invasive polymer sensors. Currently, he is Past President of the IEEE DEIS. 